\title{
Dopant enriched nitrogen gas combined with sheathless \\ CE-ESI-MS for improved sensitivity and repeatability in glycopeptide analysis
}

Guinevere S.M. Kammeijer ${ }^{1 *}$, Isabelle Kohler ${ }^{1}$, Bas C. Jansen ${ }^{1}$, Paul J. Hensbergen ${ }^{1}$, Oleg A. Mayboroda ${ }^{1}$, David Falck ${ }^{1}$, Manfred Wuhrer ${ }^{1}$

${ }^{1}$ Leiden University Medical Center, Center for Proteomics and Metabolomics, Leiden, The Netherlands

*Correspondence: Guinevere S.M. Kammeijer, Leiden University Medical Center, Center for Proteomics and Metabolomics, P.O. Box 9600, 2300 RC Leiden, The Netherlands; g.s.m.kammeijer@lumc.nl; Tel: +31-71-52-69384

\section{TABLE OF CONTENTS}

S-1. SUPPORTING INFORMATION - EXPERIMENTAL SECTION PAGE S-2

S-1.1. Tryptic digest $\quad$ PAGE S-2

S-1.2. Cotton HILIC SPE glycopeptide enrichment PAGE S-2

S-1.3. Nano-reversed phase liquid chromatography-electrospray PAGE S-3 ionization - mass spectrometry

S-1.4. Limits of detection $\quad$ PAGE S-3

S-1.5. Repeatability and intermediate precision PAGE S-3

S-1.6. Effect of electrospray flow rate PAGE S-4

S-2. SUPPORTING INFORMATION - RESULTS $\quad$ PAGE S-4

$\begin{array}{ll}\text { Table S-1 PAGE S-4 } & \text { PAGE }\end{array}$

S-3 SUPPORTING INFORMATION - TABLES S-2 - S-3 PAGE S-5

S-4 SUPPORTING INFORMATION - FIGURES S-1 - S-23 PAGE S-9

S-5 REFERENCES $\quad$ PAGE S-32 


\section{S-1. EXPERIMENTAL SECTION}

\section{S-1.1. TRYPTIC DIGEST}

Tryptic digests of polyclonal immunoglobulin $G$ (IgG), IgG subclass 1 (IgG1) and IgG monoclonal antibody 1 (IgGmAb1) were prepared in $25 \mathrm{mM} \mathrm{ABC}$ with a final concentration of $0.5 \mu \mathrm{g} / \mu \mathrm{L}$. Briefly, denaturation was performed by addition of $100 \mathrm{mM}$ FA prior to incubation for $15 \mathrm{~min}$ at room temperature, followed by evaporation to dryness at $60^{\circ} \mathrm{C}$ under vacuum for $60 \mathrm{~min}$. Dried residue was reconstituted with $25 \mathrm{mM} \mathrm{ABC}$ with a $\mathrm{pH}$ value of approximately 7.9. Overnight digestion was carried out with the enzyme trypsin from bovine pancreas, TPCK treated (Sigma-Aldrich, Steinheim, Germany) at $37^{\circ} \mathrm{C}$ (enzyme:sample 1:10, w:w).

Tryptic digests of haptoglobin ( $\mathrm{Hp}$ ) and prostate specific antigen (PSA) were prepared in $25 \mathrm{mM} \mathrm{ABC}$ with a final concentration of $0.41 \mu \mathrm{g} / \mu \mathrm{L}$ and $0.71 \mu \mathrm{g} / \mu \mathrm{L}$, respectively. Samples, originating from human serum and seminal fluid, were first diluted with $25 \mathrm{mM} \mathrm{ABC}$ to a concentration of $1 \mu \mathrm{g} / \mu \mathrm{L}$. DTT was added for reduction of the disulphide bridges at a final concentration of $2 \mathrm{mM}$ (30 min at $60^{\circ} \mathrm{C}$ ) and cooled down at room temperature. IAA was then added to the samples for alkylation at a final concentration of $6 \mathrm{mM}$ and incubated in the dark for $30 \mathrm{~min}$. DTT was added to end the sulfide alkylation at a final concentration of $6 \mathrm{mM}$. Overnight tryptic cleavage was carried out with porcine trypsin (enzyme:sample 1:30, w/w, Promega, Madison, WI ) at $37^{\circ} \mathrm{C}$. For CE-ESI-MS analysis, $\mathrm{Hp}$ and PSA tryptic digest were diluted with water to reach a concentration of $55 \mathrm{ng} / \mu \mathrm{L}$ and $18 \mathrm{ng} / \mu \mathrm{L}$, respectively, prior to further dilution with $250 \mathrm{mM} \mathrm{AAC}$ at pH 4.0 (sample:leading electrolyte 3:2, $v / v)$.

\section{S-1.2. COTTON HILIC SPE GLYCOPEPTIDE ENRICHMENT}

Glycopeptide enrichment was carried out using cotton wool hydrophilic interaction chromatography (HILIC) solid phase extraction (SPE) with a protocol modified from Selman et al. ${ }^{1}$ A 3-mm long cotton piece (Pipoos, Nieuwkuijk, Netherlands) was packed in a $20-\mu \mathrm{L}$ pipette tip (Rainin Instrument, Oakland, US). This HILIC-SPE sorbent was activated with $15 \mu \mathrm{L}$ of water prior to conditioning with a solution of $85 \% \operatorname{MeCN}(v / v)$, each step being repeated three times. Approximately $5 \mu \mathrm{g}$ of the sample dissolved in $85 \% \mathrm{MeCN}$ was loaded on the cotton wool. The glycopeptides were washed three times each with first $15 \mu \mathrm{L}$ of a solution of MeCN/water/TFA 85:14:1 $(v / v / v)$ and then $15 \mu \mathrm{L}$ of a solution of $85 \% \operatorname{MeCN}(v / v)$. Analytes were finally eluted with $10 \mu \mathrm{L}$ of water. 


\section{S-1.3. NANO-REVERSED PHASE LIQUID CHROMATOGRAPHY - ELECTROSPRAY IONIZATION - MASS}

\section{SPECTROMETRY}

Nano-reversed phase liquid chromatography (nano-LC) experiments were carried out on an UltiMate 3000 RSLCnano System (Dionex Corporation, Sunnyvale, CA), equipped with a gradient pump, a loading pump, a programmable autosampler and a column oven. Separation was carried out on a core-shell Ascentis Express C18 nanoLC column (Supelco, Bellefonte, PA; $50 \mathrm{~mm} \times 75 \mu \mathrm{m}$ i.d., 2.7 $\mu \mathrm{m}$ ), preceded by a Dionex Acclaim PepMap100 C18 trap column (Thermo Fisher Scientific Breda, the Netherlands; $5 \mathrm{~mm} \times 300 \mu \mathrm{m}$ i.d.). Mobile phase consisted of $0.1 \% \operatorname{TFA}(\mathrm{A})$ and $95 \% \operatorname{MeCN}(\mathrm{B})$, with the following gradient profile: $1 \%$ B for $0.001 \mathrm{~min}, 3-6 \% \mathrm{~B}$ in $2 \mathrm{~min}, 6-18 \% \mathrm{~B}$ in $2.5 \mathrm{~min}, 18$ $-30 \% \mathrm{~B}$ in $0.5 \mathrm{~min}$, isocratic hold at $30 \% \mathrm{~B}$ for $2 \mathrm{~min}$, back to the initial condition of $1 \% \mathrm{~B}$ in $1 \mathrm{~min}$, re-equilibration with $11 \% \mathrm{~B}$ for $3 \mathrm{~min}$. MS acquisition was switched on at $2 \mathrm{~min}$.

The nano-LC system was hyphenated to a UHR-QqTOF maXis Impact HD mass spectrometer from Bruker Daltonics (Bremen, Germany) via an electrospray ionization interface (CaptiveSprayer ${ }^{\mathrm{TM}}$ in combination with the nanoBooster ${ }^{\mathrm{TM}}$ technology from Bruker Daltonics) and MeCN as dopant, with a pressure of 0.2 bar. Experiments were carried out in ESI positive mode with a glass capillary voltage of $1200 \mathrm{~V}$ and an end plate offset voltage of $500 \mathrm{~V}$. MS source parameters were set at $3.0 \mathrm{~L} / \mathrm{min}$ for the drying gas flow rate and $180^{\circ} \mathrm{C}$ for the temperature, respectively. Quadrupole ion energy and collision cell energy were fixed at $3.0 \mathrm{eV}$ and $7.0 \mathrm{eV}$, respectively. MS data were acquired between $m / z 550-1800$ with a spectral acquisition rate of $1 \mathrm{~Hz}$. Nano-LC-ESI-MS data was analyzed with DataAnalysis 4.2 (Build 387, Bruker Daltonics).

\section{S-1.4. LIMITS OF DETECTION}

Tryptic glycopeptides of IgGmAb1 were used for the determination of the limit of detection (LOD) for nano-LC-ESI-MS and CE-ESI-MS with and without DEN-gas. LODs $(N=3)$ were determined using dilutions series, starting with a concentration of $25 \mathrm{ng} / \mu \mathrm{L}$ down to $5 \mathrm{pg} / \mu \mathrm{L}$ (5000 times diluted). For the CE-ESI-MS measurements, samples were further diluted with $250 \mathrm{mM} \mathrm{AAC}$ at $\mathrm{pH}$ 4.0 (sample: leading electrolyte, 3:2; $v / v$ ) prior to injection. The LOD was expressed as the lowest concentration where the three most abundant glycopeptides (i.e., G0F -H3N4F1-, G1F -H4N4F1and G2F -H5N4F1-) had a signal-to-noise (S/N) > 3 .

\section{S-1.5. REPEATABILITY AND INTERMEDIATE PRECISION}

Intra-day repeatability (repeatability) and inter-day repeatability (intermediate precision) were determined for the CE-ESI-MS setup equipped with the DEN-gas supply for tryptic glycopeptides of IgGmAb1 standard at three known concentrations $(c=3)$, i.e., low $(75 \mathrm{pg} / \mu \mathrm{L})$, medium $(750 \mathrm{pg} / \mu \mathrm{L})$ and high $(15 \mathrm{ng} / \mu \mathrm{L})$, on six replicates $(N=6)$ and on three consecutive days $(j=3)$. 


\section{S-1.6. EFFECT OF ELECTROSPRAY FLOW RATE}

HILIC-SPE purified IgGmAb1 glycopeptides dissolved in $10 \%$ AA were infused into the ESI-MS with flow rates between $0.9-87.9 \mathrm{~nL} / \mathrm{min}$. During infusion, the capillary voltage (in the range $950-1250$ V) was tuned at each flow rate to ensure a stable ESI current and, thus, stable MS signal.

\section{S-2. RESULTS}

Table S-1 shows the investigated ranges and optimal operating conditions for drying gas flow rate and temperature, capillary voltage, quadrupole ion energy, and collision cell energy for both conventional CE-MS and DEN-gas setup, as well as the nebulizer gas pressure for the latter. The optimal values for all the investigated parameters were found to be the same for both setups. Notably, the presence of a sheath gas within the ionization chamber does not seem to have any influence on the drying gas flow coming from the MS inlet. This may be explained by the design of the ESI source where the distance between the two gases is sufficiently large to avoid any flow perturbations. Moreover, a significant increase in peak intensities has been observed with the lowest drying gas flow rate $(1.2 \mathrm{~L} / \mathrm{min})$; lower values may lead to even better intensities but could not be tested due to software/hardware limitations. The optimal DEN-gas pressure have been set to 0.2 bar; increasing this value led to a decreased intensity.

Table S-1.Optimal ESI-MS experimental conditions with DEN-gas.

\begin{tabular}{lccc}
\hline & Investigated range & CE-ESI-MS & CE-ESI-MS with DEN-gas \\
\hline Nebulizer gas pressure [bar] & $0.1-0.3$ & - & 0.2 \\
Drying gas flow rate [L/min] & $1.2-2.5$ & 1.2 & 1.2 \\
Drying gas temperature [ $\left.{ }^{\circ} \mathrm{C}\right]$ & $150-200$ & 150 & 150 \\
Quadrupole lon Energy [eV] & $2.0-5.0$ & 3.0 & 3.0 \\
Collision Cell Energy [eV] & $4.0-10.0$ & 7.0 & 7.0 \\
\hline
\end{tabular}


Table S-2. Theoretical and observed glycopeptide mass during the CE-ESI-MS analysis of IgGmAb1 with the corresponding deviation and ppm error.

\begin{tabular}{|c|c|c|c|c|c|c|c|c|c|c|}
\hline & & & \multicolumn{8}{|c|}{ CE-ESI-MS (IgGmAb1) } \\
\hline \multicolumn{3}{|c|}{ Glycan Species* } & {$[\mathrm{M}+2 \mathrm{H}]^{2+}$} & Observed & deviation & ppm error & {$[\mathrm{M}+3 \mathrm{H}]^{3+}$} & Observed & deviation & ppm error \\
\hline 1 & H3N4F1 & $\operatorname{mov}^{7}$ & 1317.527 & 1317.524 & 0.003 & 2.277 & 878.687 & 878.6857 & 0.001 & 1.479 \\
\hline 2 & H4N4F1 & : & 1398.554 & 1398.551 & 0.003 & 2.145 & 932.705 & 932.7043 & 0.001 & 0.751 \\
\hline 3 & H5N4F1 & 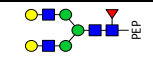 & 1479.580 & 1479.578 & 0.002 & 1.352 & 986.723 & 986.721 & 0.002 & 2.027 \\
\hline 4 & $\mathrm{H} 3 \mathrm{~N} 4$ & 10 & 1244.498 & 1244.495 & 0.003 & 2.411 & 830.001 & 829.9982 & 0.003 & 3.373 \\
\hline 5 & H4N4 & $=0$ & 1325.525 & 1325.52 & 0.005 & 3.772 & 884.019 & 884.0162 & 0.003 & 3.167 \\
\hline 6 & H5N4 & ond & 1406.551 & 1406.545 & 0.006 & 4.266 & 938.037 & 938.0332 & 0.004 & 4.051 \\
\hline 7 & H3N3F1 & $=$ & 1215.988 & 1215.984 & 0.004 & 3.290 & 810.994 & 810.9908 & 0.003 & 3.946 \\
\hline 8 & H4N3F1 & $a=$ & 1297.014 & 1297.01 & 0.004 & 3.084 & 865.012 & 865.0094 & 0.003 & 3.006 \\
\hline 9 & H3N3 & & 1142.959 & 1142.955 & 0.004 & 3.500 & 762.308 & & & \\
\hline 10 & H5N2 & & 1203.472 & 1203.468 & 0.004 & 3.324 & 802.650 & 802.6479 & 0.002 & 2.616 \\
\hline 11 & H6N2 & & 1284.498 & 1284.493 & 0.005 & 3.893 & 856.668 & 856.6663 & 0.002 & 1.984 \\
\hline 12 & H3N5F1 & go & 1419.067 & & & & & & & \\
\hline 13 & H4N5F1 & $=$ & 1500.093 & 1500.084 & 0.009 & 6.000 & 1000.398 & 1000.39 & 0.008 & 7.997 \\
\hline 14 & H5N5F1 & 물 & 1581.120 & 1581.118 & 0.002 & 1.265 & 1054.416 & 1054.406 & 0.010 & 9.484 \\
\hline 15 & H4N4F1S1 & 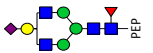 & 1544.101 & 1544.102 & -0.001 & -0.648 & 1029.737 & 1029.735 & 0.002 & 1.942 \\
\hline 16 & H5N4F1S1 & $\operatorname{rov}_{0}^{0}$ & 1625.128 & 1625.124 & 0.004 & 2.461 & 1083.754 & 1083.752 & 0.002 & 1.845 \\
\hline 17 & H5N4F1S2 & 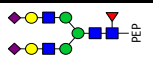 & 1770.675 & 1770.667 & 0.008 & 4.52 & 1180.786 & 1180.784 & 0.002 & 1.694 \\
\hline
\end{tabular}

* Glycan compositions are given in terms of number of hexoses $(\mathrm{H}), \mathrm{N}$-acetylglucosamines (N), fucoses (F) and sialic acids (S). 
Table S-3. Theoretical and observed glycopeptide mass during the CE-ESI-MS analysis with DEN-gas of IgGmAb1 with the corresponding deviation and ppm error

\begin{tabular}{|c|c|c|c|c|c|c|c|c|c|c|c|c|c|c|}
\hline & & & \multicolumn{12}{|c|}{ CE-ESI-MS with DEN-gas (IgGmAb1) } \\
\hline \multicolumn{3}{|c|}{ Glycan Species* } & {$[\mathrm{M}+2 \mathrm{H}]^{2+}$} & Observed & deviation & ppm error & {$[\mathrm{M}+3 \mathrm{H}]^{3+}$} & Observed & deviation & ppm error & {$[M+4 H]^{4+}$} & Observed & deviation & ppm error \\
\hline 1 & H3N4F1 & प0. & 1317.527 & 1317.52 & 0.007 & 5.31 & 878.687 & 878.6857 & 0.001 & 1.48 & 659.268 & 659.2603 & 0.008 & 11.68 \\
\hline 2 & H4N4F1 & 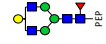 & 1398.554 & 1398.546 & 0.008 & 5.72 & 932.705 & 932.7043 & 0.001 & 0.75 & 699.781 & 699.7775 & 0.003 & 5.00 \\
\hline 3 & H5N4F1 & Grit & 1479.580 & 1479.572 & 0.008 & 5.41 & 986.723 & 986.721 & 0.002 & 2.03 & 740.294 & 740.2907 & 0.003 & 4.46 \\
\hline 4 & $\mathrm{H} 3 \mathrm{~N} 4$ & -0 & 1244.498 & 1244.491 & 0.007 & 5.62 & 830.001 & 829.9982 & 0.003 & 3.37 & 622.753 & & & \\
\hline 5 & $\mathrm{H} 4 \mathrm{~N} 4$ & .0. & 1325.525 & 1325.516 & 0.009 & 6.79 & 884.019 & 884.0162 & 0.003 & 3.17 & 663.266 & 663.2611 & 0.005 & 7.39 \\
\hline 6 & H5N4 & 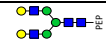 & 1406.551 & 1406.537 & 0.014 & 9.95 & 938.037 & 938.0332 & 0.004 & 4.05 & 703.780 & 703.7752 & 0.005 & 6.82 \\
\hline 7 & H3N3F1 & - $8=1$ & 1215.988 & 1215.98 & 0.008 & 6.58 & 810.994 & 810.9908 & 0.003 & 3.95 & 608.498 & & & \\
\hline 8 & H4N3F1 & 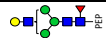 & 1297.014 & 1297.006 & 0.008 & 6.17 & 865.012 & 865.0094 & 0.003 & 3.01 & 649.011 & & & \\
\hline 9 & H3N3 & & 1142.959 & 1142.953 & 0.006 & 5.25 & 762.308 & & & & 571.983 & & & \\
\hline 10 & $\mathrm{H} 5 \mathrm{~N} 2$ & -t魚 & 1203.472 & 1203.465 & 0.007 & 5.82 & 802.650 & 802.6479 & 0.002 & 2.62 & 602.240 & & & \\
\hline 11 & $\mathrm{H} 6 \mathrm{~N} 2$ & $20=\frac{1}{4}$ & 1284.498 & 1284.491 & 0.007 & 5.45 & 856.668 & 856.6663 & 0.002 & 1.98 & 642.753 & & & \\
\hline 12 & H3N5F1 & 9-8) & 1419.067 & 1419.051 & 0.016 & 11.28 & 946.381 & & & & 710.037 & & & \\
\hline 13 & H4N5F1 & 98 & 1500.093 & 1500.075 & 0.018 & 12.00 & 1000.398 & 1000.39 & 0.008 & 8.00 & 750.551 & & & \\
\hline 14 & H5N5F1 & 90 & 1581.120 & 1581.105 & 0.015 & 9.49 & 1054.416 & 1054.406 & 0.010 & 9.48 & 791.064 & & & \\
\hline 15 & H4N4F1S1 & 焉 & 1544.101 & 1544.088 & 0.013 & 8.42 & 1029.737 & 1029.735 & 0.002 & 1.94 & 772.555 & 772.5506 & 0.004 & 5.70 \\
\hline 16 & H5N4F1S1 & 4 & 1625.128 & 1625.111 & 0.017 & 10.46 & 1083.754 & 1083.752 & 0.002 & 1.85 & 813.068 & 813.0664 & 0.002 & 1.97 \\
\hline 17 & H5N4F1S2 & $+0 \log ^{-1}$ & 1770.675 & 1770.658 & 0.017 & 9.60 & 1180.786 & 1180.784 & 0.002 & 1.69 & 885.842 & 885.8426 & -0.001 & -0.68 \\
\hline
\end{tabular}

* Glycan compositions are given in terms of number of hexoses (H), $N$-acetylglucosamines (N), fucoses (F) and sialic acids (S). 
Table S-4. Theoretical and observed glycopeptide mass during the CE-ESI-MS analysis of Hp with the corresponding deviation and ppm error.

\begin{tabular}{|c|c|c|c|c|c|c|c|c|c|c|c|}
\hline & & & \multicolumn{9}{|c|}{ N184 - PEP = MVSHHNLTTGATLINEQWLLTTAK } \\
\hline \multicolumn{3}{|c|}{ Glycan Species* } & {$[\mathrm{M}+4 \mathrm{H}]^{4+}$} & Observed & deviation & ppm error & {$[M+5 H]^{5+}$} & Observed & deviation & ppm error & MS2* \\
\hline 1 & H5N4S1 & - :- $=0$ & 1149.023 & 1149.03 & -0.007 & -6.09 & 919.420 & 919.4248 & -0.005 & -5.22 & $x$ \\
\hline 2 & H5N4F1S1 & $-x^{2}$ & 1185.538 & 1185.545 & -0.007 & -5.90 & 948.632 & 948.6351 & -0.003 & -3.27 & \\
\hline 3 & H5N4S2 & $\bullet$ & 1221.797 & 1221.804 & -0.007 & -5.73 & 977.639 & 977.6421 & -0.003 & -3.17 & $x$ \\
\hline 4 & H5N4F1S2 & 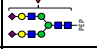 & 1258.312 & 1258.318 & -0.006 & -4.77 & 1006.851 & 1006.855 & -0.004 & -3.97 & \\
\hline 5 & H6N5S1 & 0 & 1240.306 & 1240.316 & -0.010 & -8.06 & 992.447 & 992.4557 & -0.009 & -8.77 & \\
\hline 6 & H6N5S2 & $:-1=$ & 1313.080 & 1313.085 & -0.005 & -3.81 & 1050.666 & 1050.669 & -0.003 & -2.86 & $x$ \\
\hline 7 & H6N5S3 & - & 1385.854 & 1385.861 & -0.007 & -5.05 & 1108.885 & 1108.891 & -0.006 & -5.41 & $x$ \\
\hline 8 & HENSF1S3 & $0=1$ & 1422.369 & 1422.371 & -0.002 & -1.41 & 1138.097 & 1138.103 & -0.006 & -5.27 & \\
\hline
\end{tabular}

\begin{tabular}{|c|c|c|c|c|c|c|c|c|c|c|c|c|c|c|c|}
\hline & & & \multicolumn{13}{|c|}{ N207-211 - PEP = NLFLNHSENATAK } \\
\hline \multicolumn{3}{|c|}{ Glycan Species } & {$[M+3 H]^{3+}$} & Observed & deviation & ppm error & {$[M+4 H] 4^{+}$} & Observed & deviation & ppm error & {$[M+5 H]^{5+}$} & Observed & deviation & ppm error & MS2* \\
\hline 1 & H5N4S1 & $\cdot 0=1$ & 1124.809 & 1124.814 & -0.005 & -4.45 & 843.859 & 843.8568 & 0.002 & 2.61 & 675.289 & & & & $x$ \\
\hline 2 & H5N4S2 & $-0=\tilde{x}$ & 1221.841 & & & & 916.633 & 916.6362 & -0.003 & -3.49 & 733.508 & & & & $x$ \\
\hline 3 & H10N8S2 & & 1762.701 & 1762.711 & -0.010 & -5.67 & 1322.278 & 1322.28 & -0.002 & -1.51 & 1058.024 & 1058.028 & -0.004 & -3.78 & $\mathrm{x}$ \\
\hline 4 & H10N8S3 & & 1859.733 & & & & 1395.052 & 1395.057 & -0.005 & -3.58 & 1116.243 & 1116.249 & -0.006 & -5.38 & $x$ \\
\hline 5 & H10N8S4 & & 1956.765 & & & & 1467.826 & 1467.832 & -0.006 & -4.09 & 1174.462 & 1174.471 & -0.009 & -7.66 & \\
\hline 6 & H11N9S2 & & 1884.412 & 1884.427 & -0.015 & -7.96 & 1413.561 & 1413.572 & -0.011 & -7.78 & 1131.050 & 1131.056 & -0.006 & -5.30 & $x$ \\
\hline 7 & H11N9S3 & & 1981.444 & & & & 1486.335 & 1486.341 & -0.006 & -4.04 & 1189.270 & 1189.279 & -0.009 & -7.57 & $\mathrm{x}$ \\
\hline 8 & H11N9S4 & & 2078.476 & & & & 1559.109 & 1559.122 & -0.013 & -8.34 & 1247.489 & 1247.5 & -0.011 & -8.82 & \\
\hline & & & \multicolumn{13}{|c|}{ N241 - PEP = VVLHPNYSQVDIGLIK } \\
\hline \multicolumn{3}{|c|}{ Glycan Species } & {$[M+3 H]^{3+}$} & Observed & deviation & ppm error & {$[M+4 H] 4^{+}$} & Observed & deviation & ppm error & {$[M+5 H]^{5+}$} & Observed & deviation & ppm error & MS2* \\
\hline 1 & H5N4S2 & $-0=-2$ & 1333.933 & 1333.939 & -0.006 & -4.50 & 1000.702 & 1000.709 & -0.007 & -7.00 & 800.763 & 800.7685 & -0.005 & -6.87 & $x$ \\
\hline 2 & H5N4S1 & $+0-2$ & 1236.902 & 1236.909 & -0.007 & -5.66 & 927.928 & 927.9312 & -0.003 & -3.45 & 742.544 & & & & $x$ \\
\hline 3 & H6N5S1 & $\cdot 0$ & 1358.612 & & & & 1019.211 & 1019.217 & -0.006 & -5.89 & 815.571 & & & & $x$ \\
\hline 4 & H6N5S2 & $: \because$ & 1455.644 & 1455.65 & -0.006 & -4.12 & 1091.985 & 1091.993 & -0.008 & -7.33 & 873.790 & 873.7914 & -0.001 & -1.60 & $\mathrm{x}$ \\
\hline 5 & H7NGS2 & $\because:$ & 1577.355 & 1577.362 & -0.007 & -4.44 & 1183.268 & 1183.278 & -0.010 & -8.45 & 946.816 & 946.8249 & -0.009 & -9.40 & $x$ \\
\hline 6 & H6N5S3 & - & 1552.676 & 1552.681 & -0.005 & -3.22 & 1164.759 & 1164.766 & -0.007 & -6.01 & 932.009 & 932.0133 & -0.004 & -4.61 & $x$ \\
\hline 7 & H6N5F1S3 & 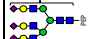 & 1601.362 & 1601.371 & -0.009 & -5.62 & 1201.273 & 1201.281 & -0.008 & -6.66 & 961.220 & 961.223 & -0.003 & -3.12 & $x$ \\
\hline 8 & H7N6S3 & $1-\hat{\mathbb{R}}$ & 1674.387 & 1674.396 & -0.009 & -5.38 & 1256.042 & 1256.051 & -0.009 & -7.17 & 1005.035 & 1005.043 & -0.008 & -7.96 & $x$ \\
\hline
\end{tabular}

* Glycan compositions are given in terms of number of hexoses $(\mathrm{H}), \mathrm{N}$-acetylglucosamines $(\mathrm{N})$, fucoses $(\mathrm{F})$ and sialic acids $(\mathrm{S})$.

$X$ confirmed with MS2

Selected mass is used for the MS2 confirmation 
Table S-5. Theoretical and observed glycopeptide mass during the CE-ESI-MS analysis of PSA with the corresponding deviation and ppm error.

\begin{tabular}{|c|c|c|c|c|c|c|c|c|c|c|c|}
\hline & & & \multicolumn{9}{|c|}{ N69 - PEP $=\mathbf{N K}$} \\
\hline \multicolumn{3}{|c|}{ Glycan Species* } & {$[M+4 H]^{4+}$} & Observed & deviation & ppm error & {$[M+5 H]^{5+}$} & Observed & deviation & ppm error & MS2* \\
\hline 1 & H4N3F1S1 & - & 978.384 & 978.3816 & 0.002 & 1.95 & 652.592 & & & & $x$ \\
\hline 2 & H4N4F1S1 & -Q Go & 1079.923 & 1079.925 & -0.002 & -1.67 & 720.285 & 720.2826 & 0.002 & 3.02 & \\
\hline 3 & H5N4S1 & - & 1087.921 & 1087.922 & -0.001 & -1.24 & 725.616 & 725.6144 & 0.002 & 2.78 & \\
\hline 4 & H5N4F1S1 & - $\cos ^{7}$ & 1160.950 & 1160.954 & -0.004 & -3.78 & 774.302 & 774.3001 & 0.002 & 2.95 & $x$ \\
\hline 6 & H4N5F1S1 & - & 1181.463 & 1181.466 & -0.003 & -2.64 & 787.978 & 787.9762 & 0.002 & 2.16 & \\
\hline 7 & H5N4S2 & - $0^{\circ}=\frac{1}{2}$ & 1233.468 & 1233.469 & -0.001 & -0.52 & 822.648 & 822.6457 & 0.003 & 3.06 & $x$ \\
\hline 8 & H5N4F1S2 & - & 1306.497 & 1306.491 & 0.006 & 4.83 & 871.334 & 871.3322 & 0.002 & 2.28 & $x$ \\
\hline 9 & H4N5S2 & - & 1253.982 & 1253.981 & 0.001 & 0.51 & 836.324 & 836.3209 & 0.003 & 3.39 & \\
\hline 10 & H4N5F1S2 & Gon & 1327.011 & 1327.006 & 0.005 & 3.46 & 885.010 & 885.0072 & 0.003 & 2.83 & $x$ \\
\hline
\end{tabular}

* Glycan compositions are given in terms of number of hexoses (H), $\mathrm{N}$-acetylglucosamines (N), fucoses (F) and sialic acids (S).

$X$ confirmed with MS2

Selected mass is used for the MS2 confirmation 

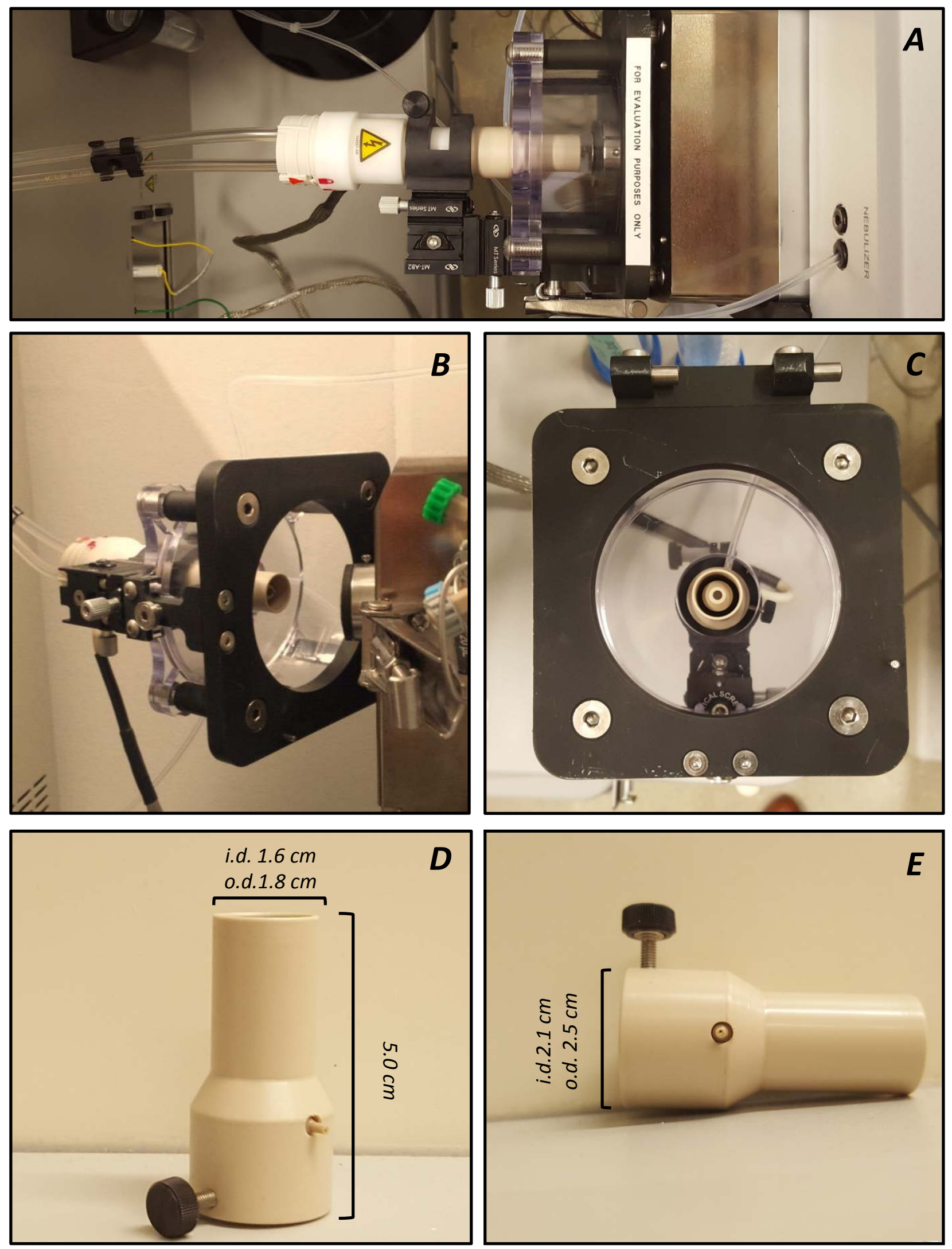

Figure S-1. CE-ESI-MS with DEN-gas setup using an in-house made cover. (A) Top view from the source in front of the mass spectrometer, the in-house made cover is slid onto the CE-ESI capillary cartridge. (B) Side view of the ionization chamber. (C) Front view of the ionization chamber. (D) In-house made cover with inner diameter (i.d.) and outer diameter (o.d.) displayed for lateral and front parts. (E) In-house made cover with inner diameter (i.d.) and outer diameter (o.d.) displayed for back part. 

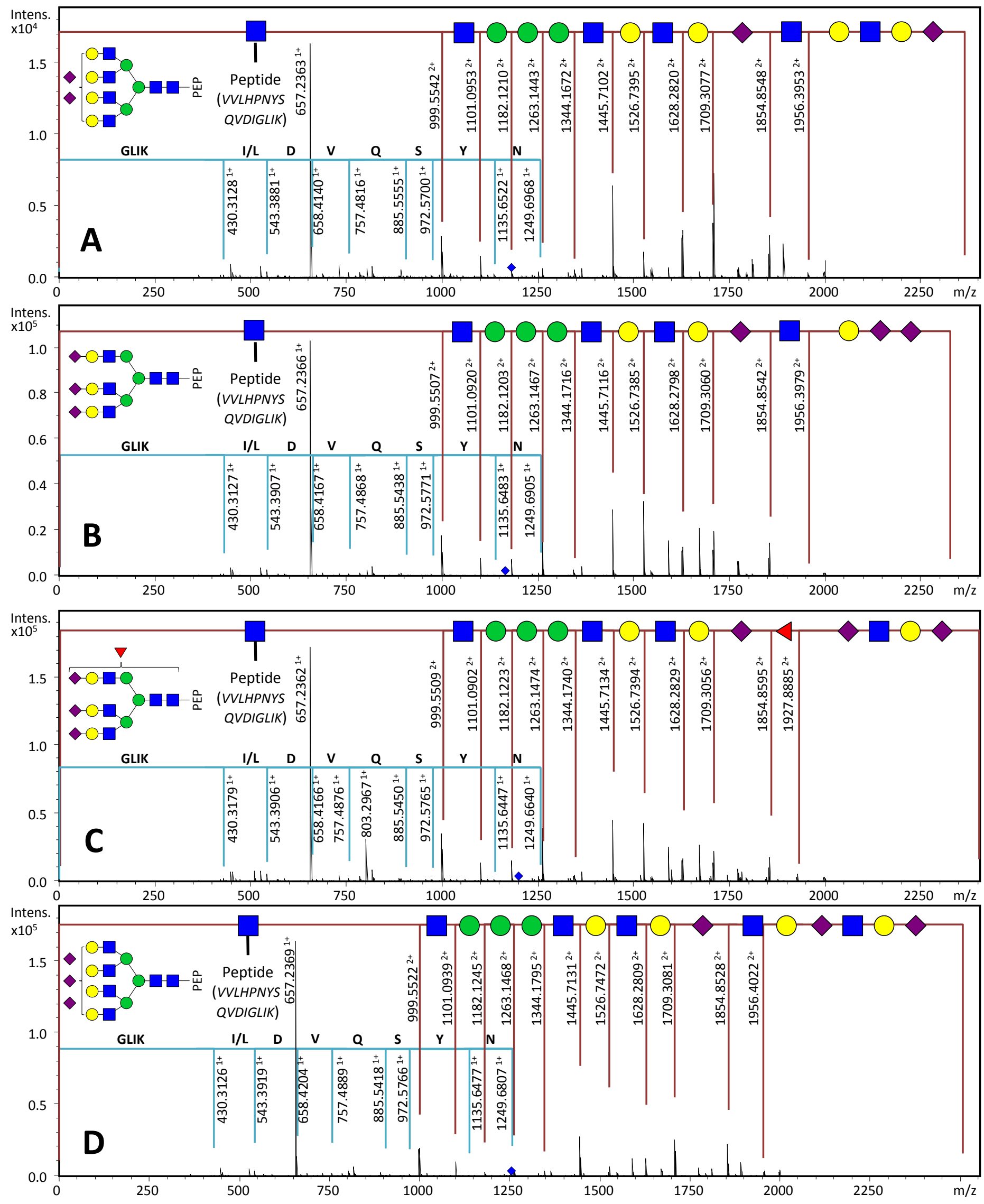

Mannose $\bigcirc$ Galactose $\square$-Acetylglucosamine

N-Acetylgalactosamine $\triangle$ Fucose

$N$-Acetylneuraminic acid

Figure S-4. Fragmentation spectra of high abundant tryptic glycopeptides at glycosylation site $\mathbf{N}_{241}$ from $\mathrm{Hp}$ analysed with CE-ESI-MS using DEN-gas. The following precursor ions were fragmented (A) $1183.7700^{4+}$ (H7N6S2), (B) $1165.5171{ }^{4+}$ (H6N5S3) (C) $1202.0254{ }^{4+}$ (H6N5F1S3), (D) $1256.5554{ }^{5+}$ (H7N6S3). Blue diamond illustrates the precursor ion, PEP illustrates the tryptic peptide sequence MVSHHNLTTGATLINEQWLLTTAK. 

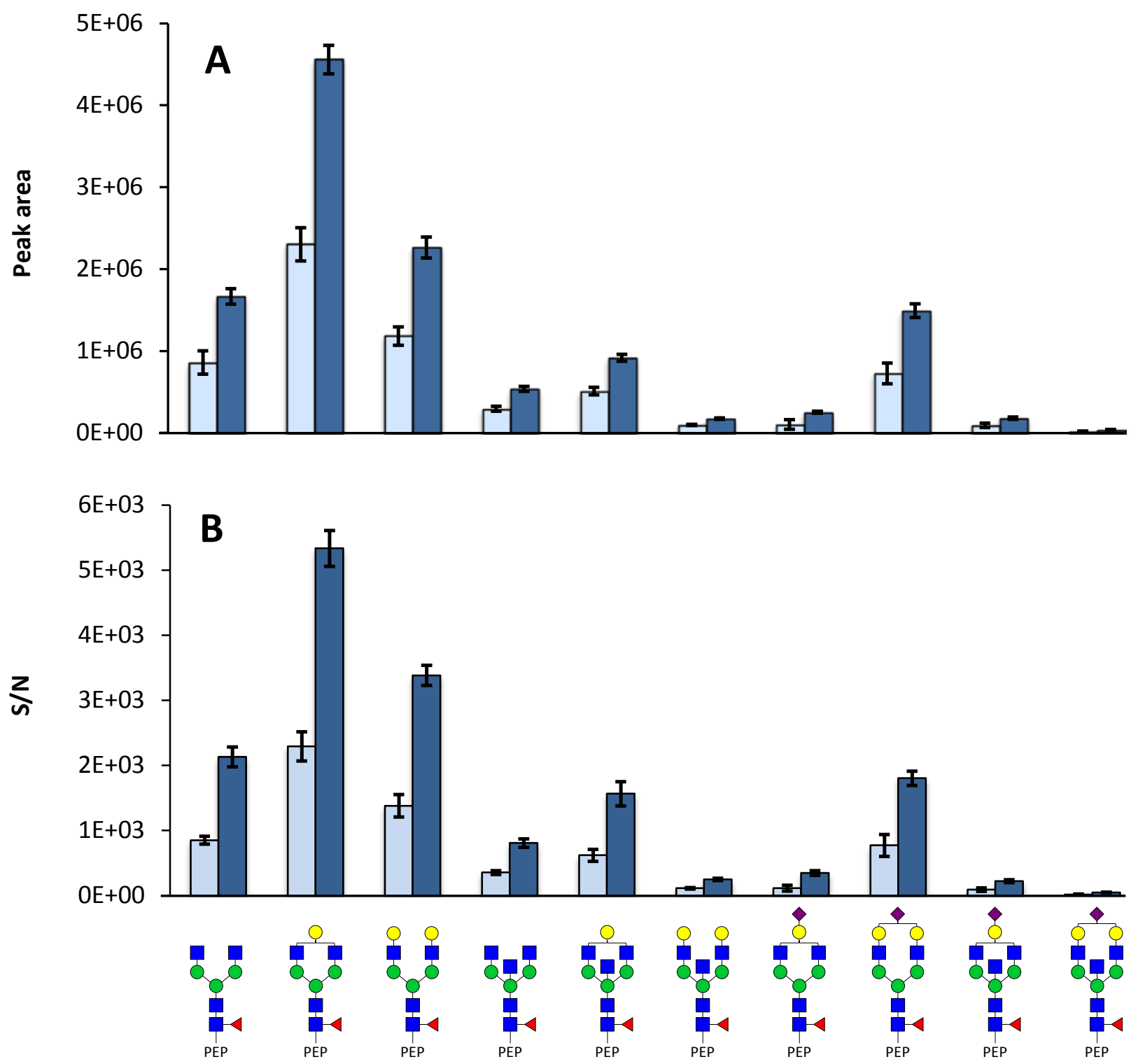

Figure S-8. Differences in peak areas and signal-to-noise ratio $(\mathrm{S} / \mathrm{N})$ observed for the tryptic fucosylated glycopeptides from polyclonal antibody IgG subclass 1 between the CE-ESI-MS (light blue) and CE-ESI-MS with DEN-gas setup (dark blue). (A) absolute peak areas and (B) $\mathrm{S} / \mathrm{N}$ ratios. Peak areas and $\mathrm{S} / \mathrm{N}$ ratios were measured on EIE. Error bars represent the standard deviation $(N=3)$. PEP illustrates the tryptic peptide sequence EEQYNSTYR. 


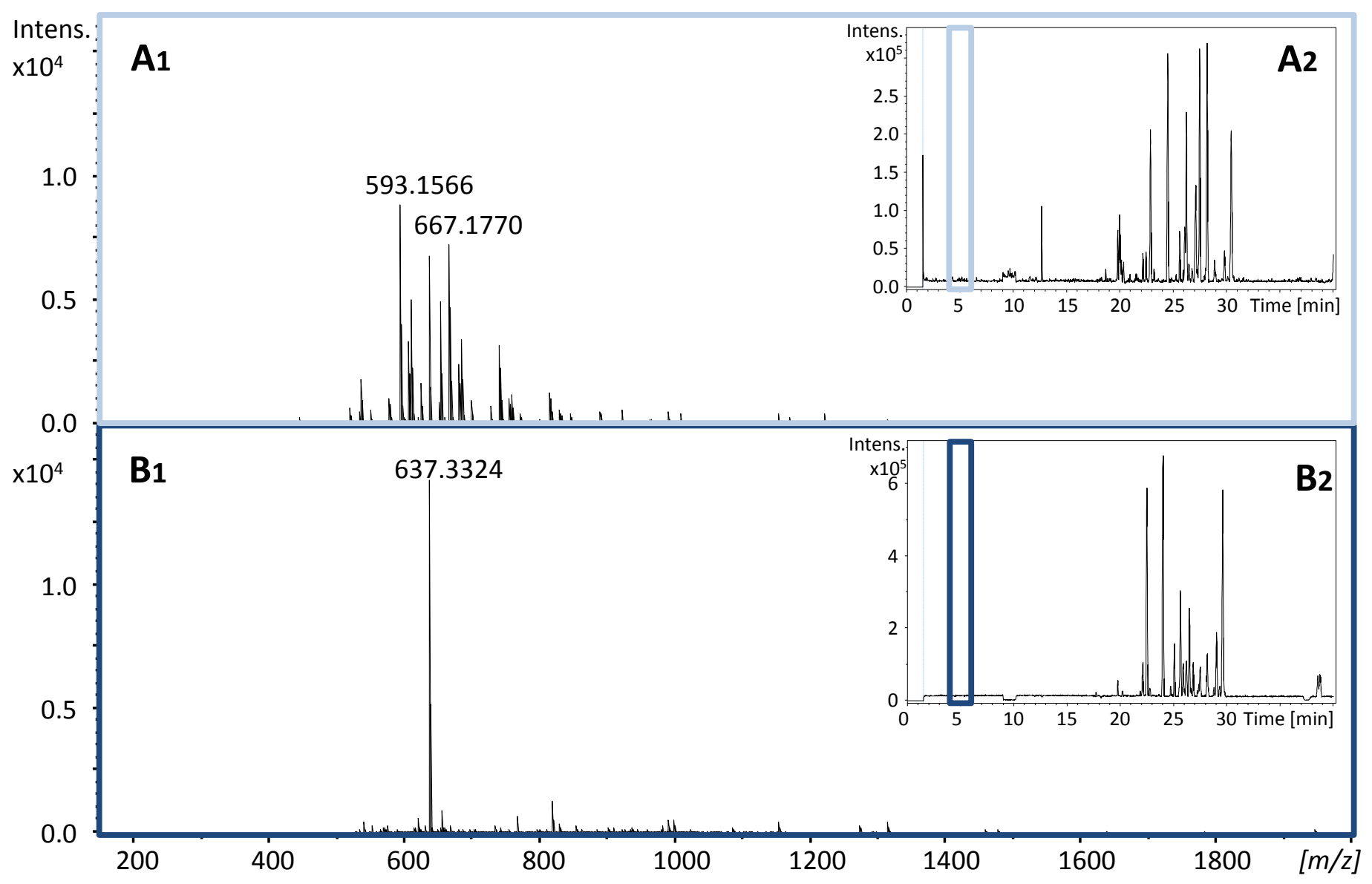

Figure S-9. Mass spectra (sum spectra) recorded between 4.5 and 5.5 min during analysis of tryptic glycopeptides from polyclonal antibody IgG, corresponding to the background signal. (A1) MS spectrum obtained with conventional CE-ESI-MS setup , (B1) MS spectrum obtained with CE-ESI-MS using DEN-gas. A2 and B2 are the base peak electropherograms of the analysis with an indication in the analysis where the summed spectra was recorded. 

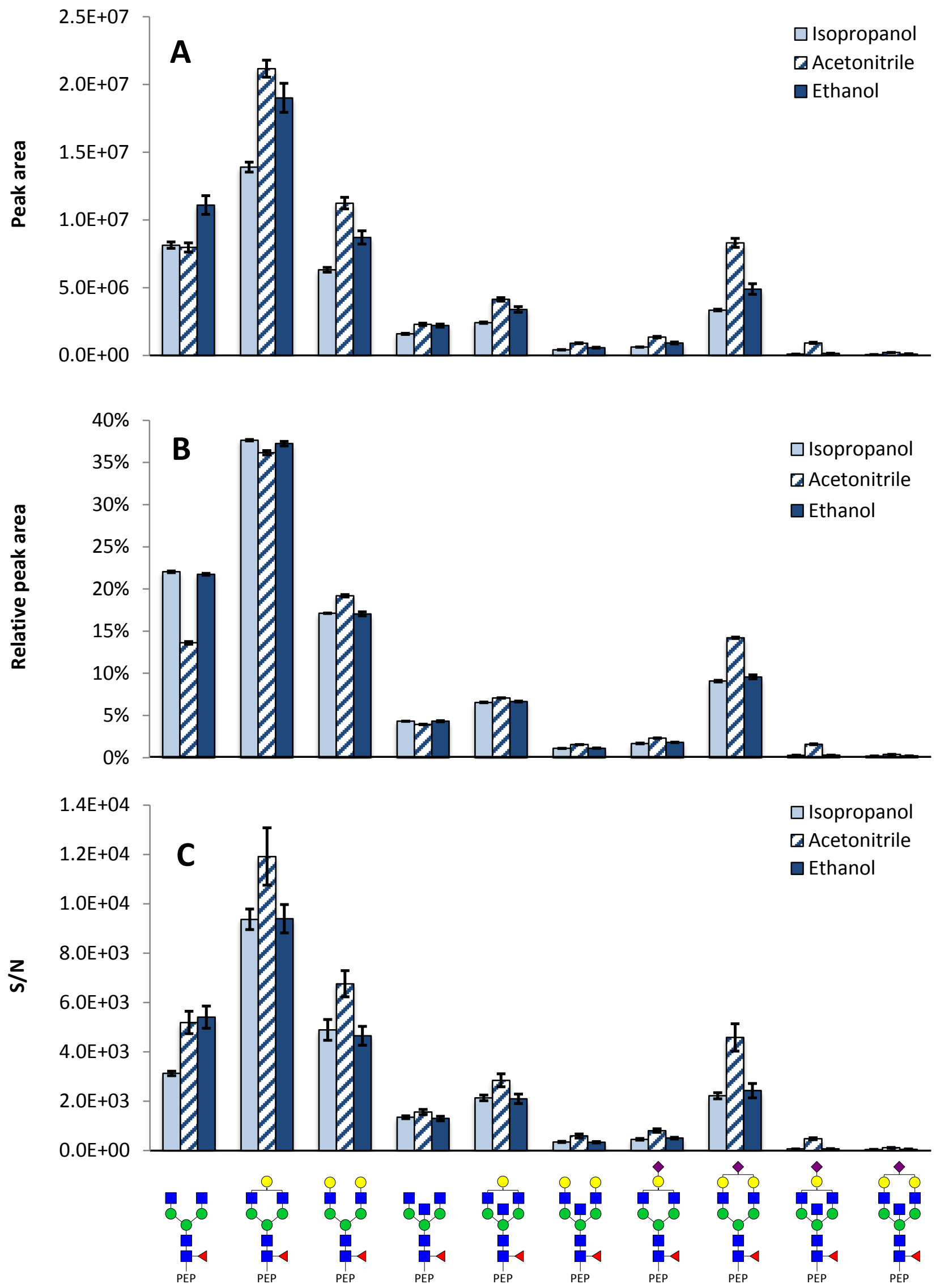

Figure S-10. Effect of solvent used for the DEN-gas on peak areas from fucosylated glycopeptides of polyclonal antibody IgG subclass 1 glycopeptides. (A) Absolute peak areas, (B) relative peak areas and (C) S/N ratios. Peak areas were measured on EIE. Error bars represent the standard deviation $(N=3)$. PEP illustrates the tryptic peptide sequence EEQYNSTYR. 

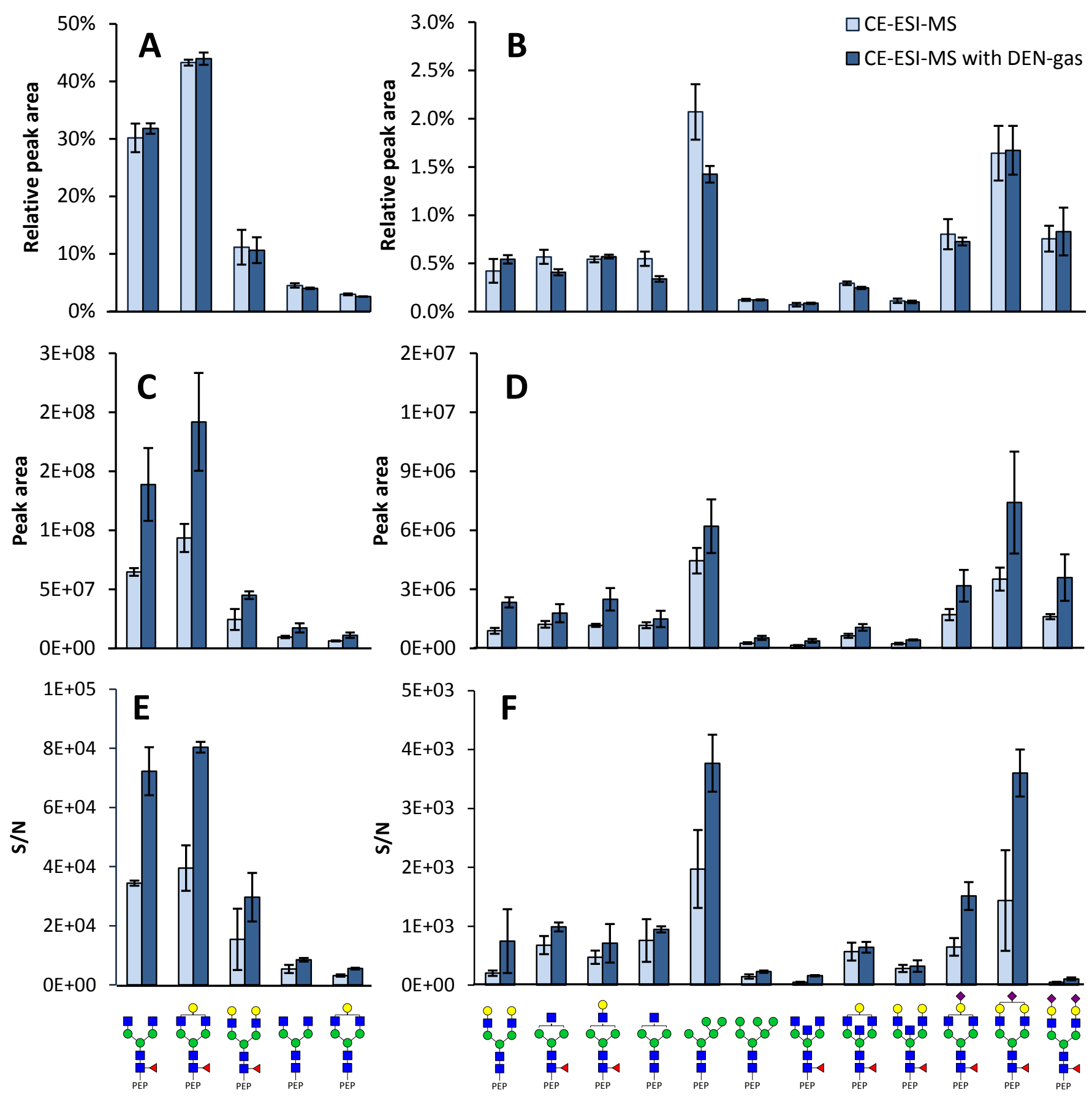

Figure S-11. Differences in peak area and signal-to-noise ratio $(\mathrm{S} / \mathrm{N})$ observed for the tryptic Fc $\mathrm{N}$ glycopeptides from IgGmAb1 between conventional CE-ESI-MS (light blue) and CE-ESI-MS with DEN-gas setup (dark blue). (A) Relative peak areas of the most abundant glycopeptides, (B) relative peak areas of the low abundant glycopeptides. (C) Absolute peak areas of the most abundant glycopeptides, (D) absolute peak areas of the low abundant glycopeptides. (E) S/N ratios of the most abundant glycopeptides, (F) S/N ratios of the low abundant glycopeptides. Peak areas and $\mathrm{S} / \mathrm{N}$ ratios were measured on EIE. Error bars represent the standard deviation $(N=3)$. PEP illustrates the tryptic peptide sequence EEQYNSTYR. 

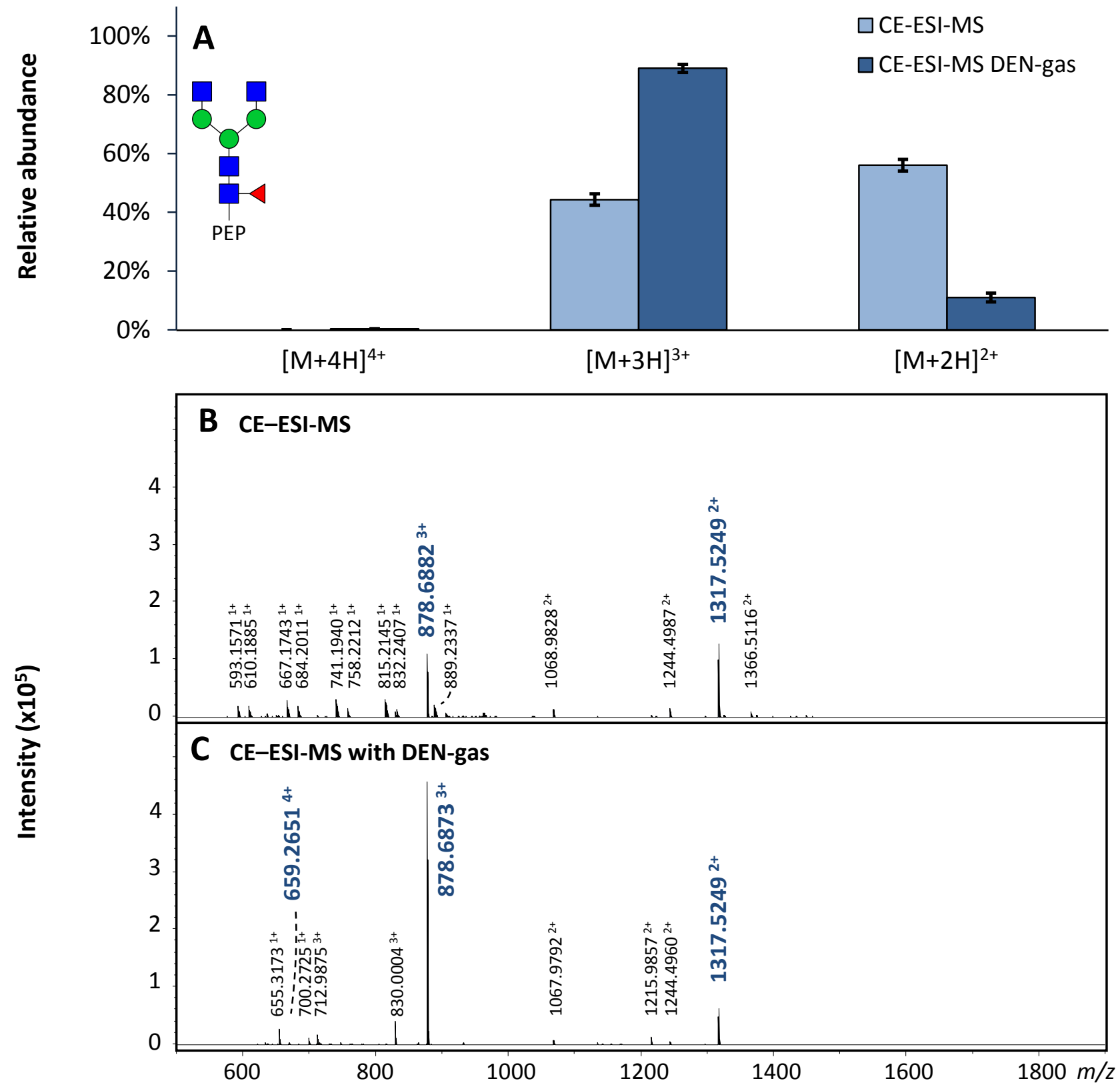

Figure S-12. Summed spectra recorded between $\mathbf{2 6 . 0}$ and $\mathbf{2 7 . 5} \mathrm{min}$ corresponding to the migration of the tryptic Fc N-glycopeptide GOF from IgGmAb1. (A) Relative abundance of doubly, triply and quadruply charged species observed during CE-ESI-MS analysis with (dark blue) and without (light blue) DEN-gas $(N=3)$. (B) MS spectrum obtained with CEESI-MS setup without DEN-gas, (C) MS spectrum obtained with CE-ESI-MS using DEN-gas. PEP illustrates the tryptic peptide sequence EEQYNSTYR. 

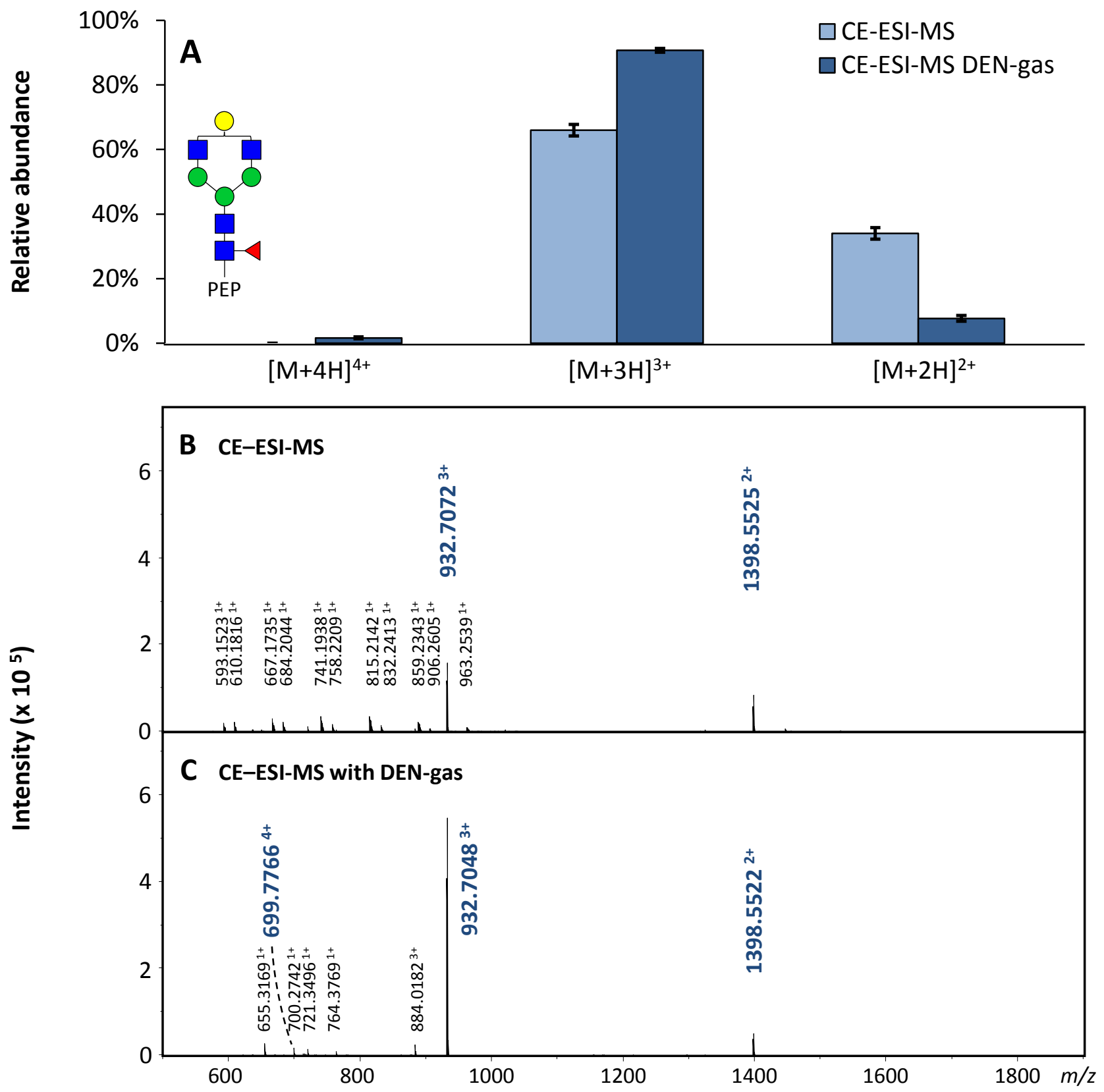

Figure S-13. Summed spectra recorded between $\mathbf{2 6 . 3}$ and $\mathbf{2 7 . 8} \mathrm{min}$ corresponding to the migration of the tryptic Fc N-glycopeptide G1F from IgGmAb1. (A) Relative abundance of doubly, triply and quadruply charged species observed during CE-ESI-MS analysis with (dark blue) and without (light blue) DEN-gas $(N=3)$. (B) MS spectrum obtained with CEESI-MS setup without DEN-gas, (C) MS spectrum obtained with CE-ESI-MS using DEN-gas. PEP illustrates the tryptic peptide sequence EEQYNSTYR. 

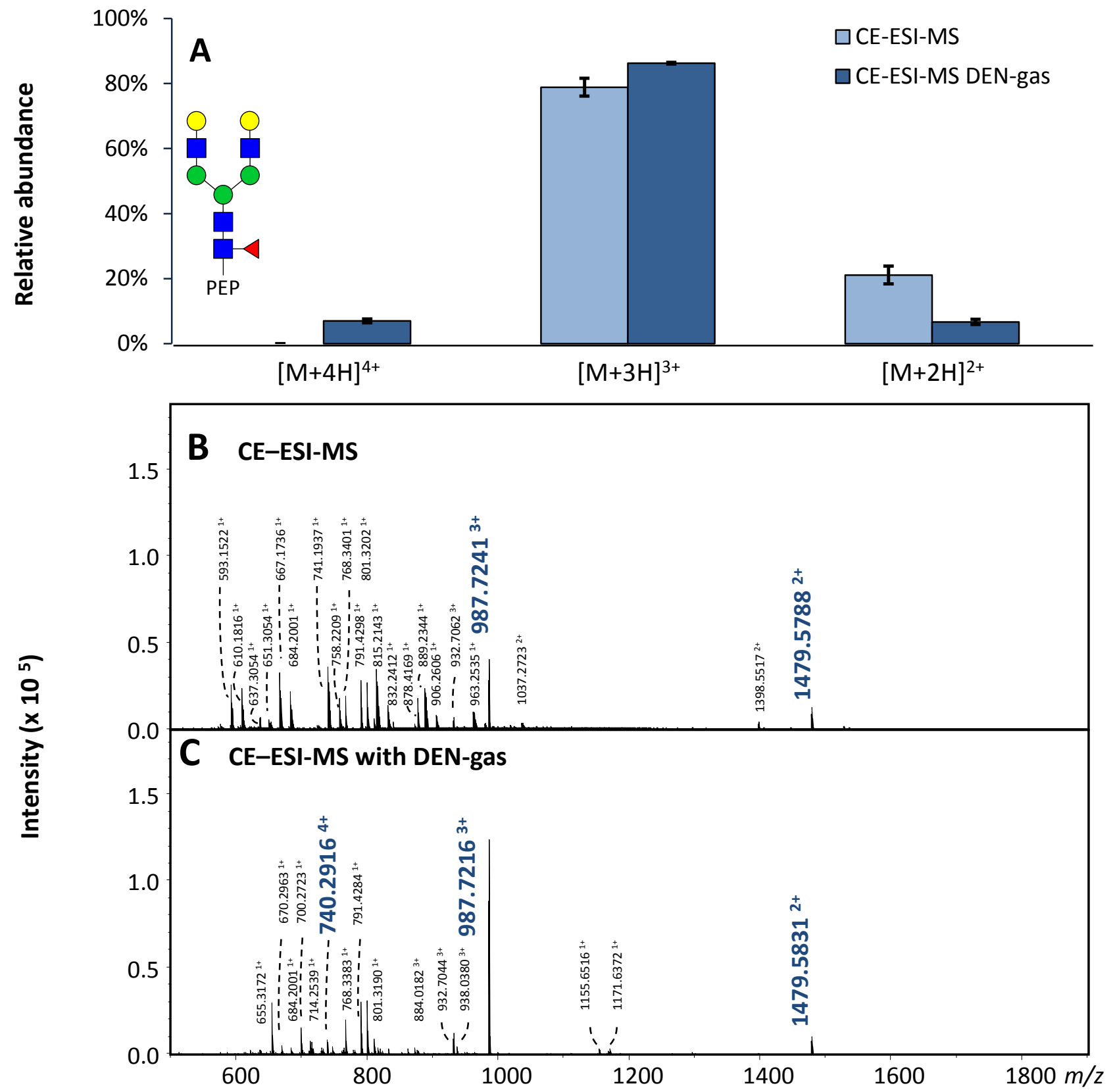

Figure S-14. Summed spectra recorded between 26.4 and $28.0 \mathrm{~min}$ corresponding to the migration of the tryptic Fc N-glycopeptide G2F from IgGmAb1. (A) Relative abundance of doubly, triply and quadruply charged species observed during CE-ESI-MS analysis with (dark blue) and without (light blue) DEN-gas $(N=3)$. (B) MS spectrum obtained with CEESI-MS setup without DEN-gas, (C) MS spectrum obtained with CE-ESI-MS using DEN-gas. PEP illustrates the tryptic peptide sequence EEQYNSTYR. 


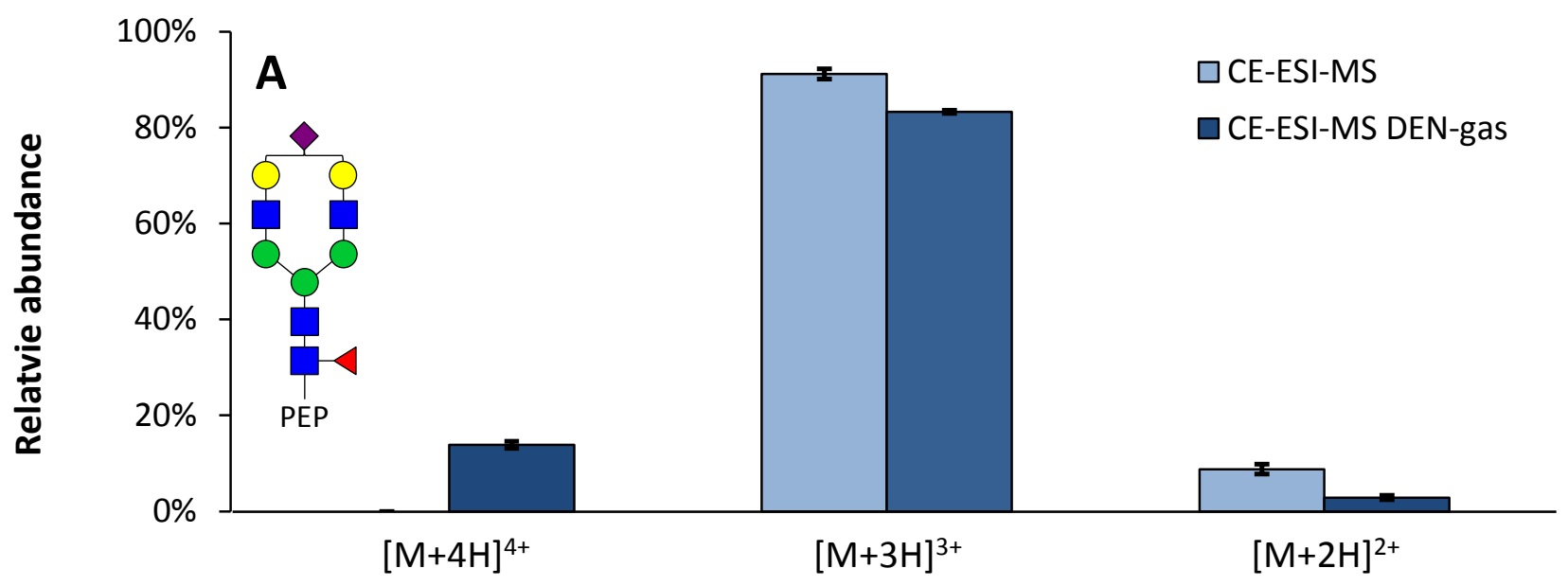

\section{B CE-ESI-MS}

4

C CE-ESI-MS with DEN-gas

$$
\begin{aligned}
& + \\
& \infty \\
& \infty \\
& N \\
& 0 \\
& \infty \\
& \infty
\end{aligned}
$$

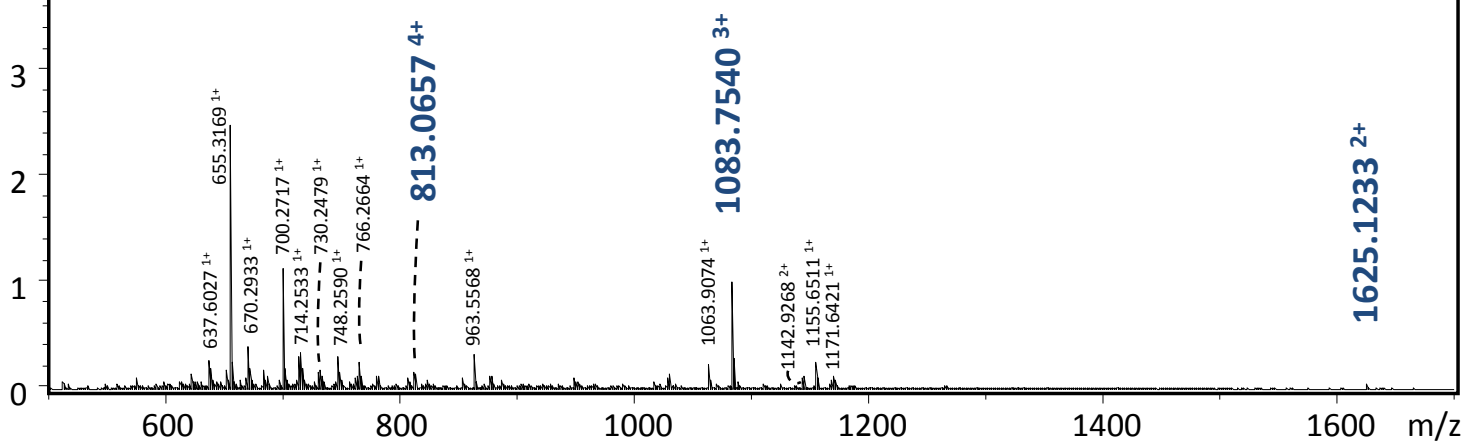

Figure S-15. Summed spectra recorded between 29.3 and $31.7 \mathrm{~min}$ corresponding to the migration of the tryptic Fc $\mathrm{N}$-glycopeptide G2FS from IgGmAb1. (A) Relative abundance of doubly, triply and quadruply charged species observed during CE-ESI-MS analysis with (dark blue) and without (light blue) DEN-gas $(N=3)$. (B) MS spectrum obtained with CEESI-MS setup without DEN-gas, (C) MS spectrum obtained with CE-ESI-MS using DEN-gas. PEP illustrates the tryptic peptide sequence EEQYNSTYR. 


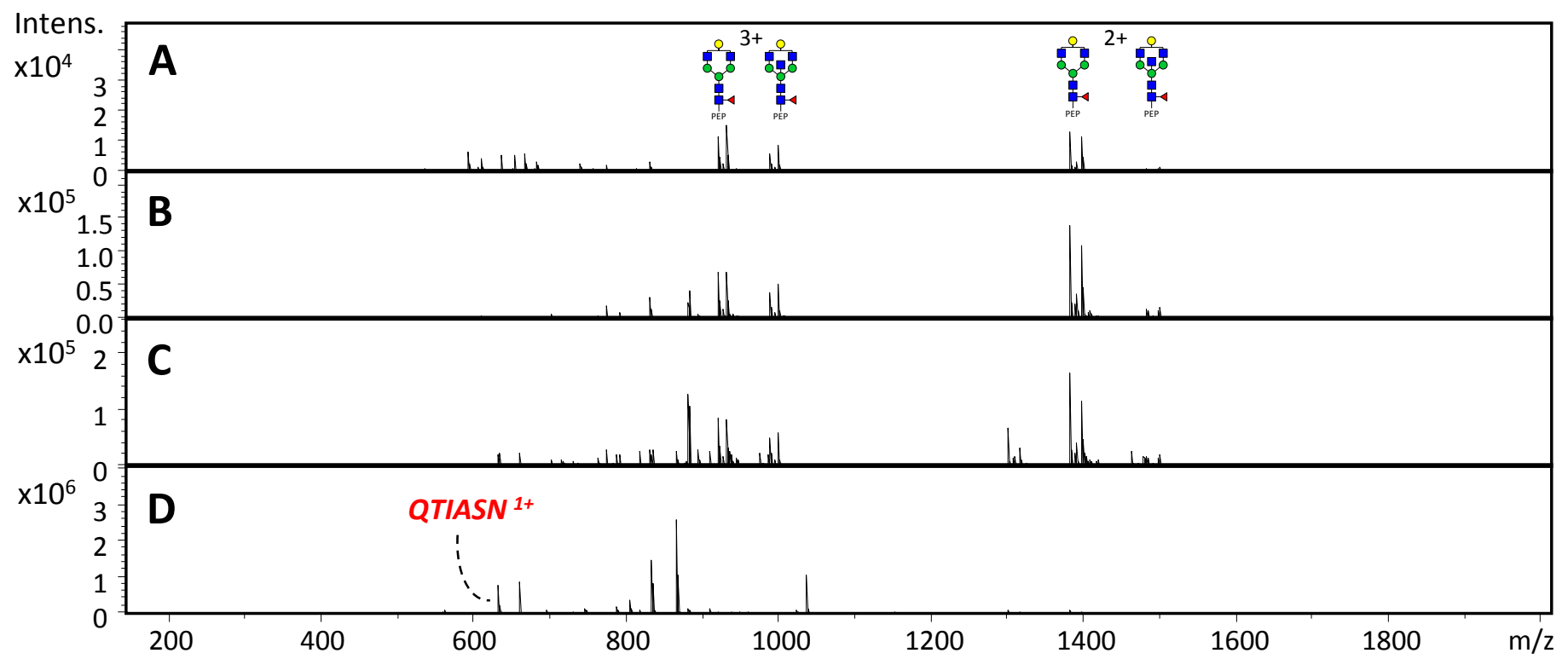

Figure S-17.1. Summed spectra recorded between 22.7 and $27.0 \mathrm{~min}$ corresponding to the migration of the tryptic non-sialylated Fc $\mathbf{N}$-glycopeptides from polyclonal IgG with different injection volumes using conventional CE-ESI-MS. (A) Injection of $8.8 \mathrm{~nL}$, corresponding to $1 \%$ of the capillary volume, (B) injection of $114 \mathrm{~nL}$, corresponding to $18 \%$ of the capillary volume, (C) injection of $220 \mathrm{~nL}$, corresponding to $35 \%$ of the capillary volume, (D) injection of $352 \mathrm{~nL}$, corresponding to $55 \%$ of the capillary volume. The MS spectra show the co-migration of glycopeptides of interest with an autolysis product from bovine trypsin with peptide sequence (pep.seq) QTIASN. PEP illustrates the tryptic peptide sequence EEQYNSTYR.

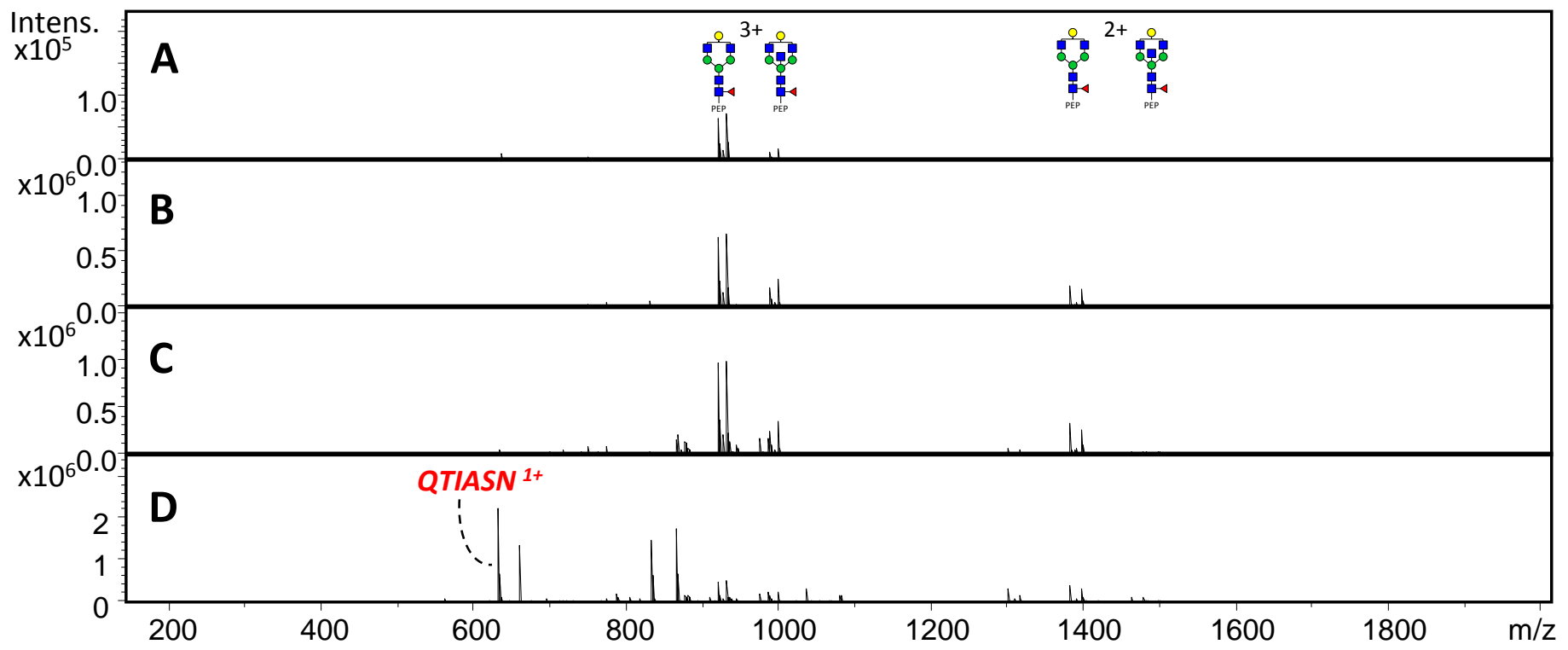

Figure S-17.2. Summed spectra recorded between 23.5 and $26.5 \mathrm{~min}$ corresponding to the migration of the tryptic non-sialylated Fc $\mathrm{N}$-glycopeptides from polyclonal IgG with different injection volumes using CE-ESI-MS with DEN-gas. (A) Injection of $8.8 \mathrm{~nL}$, corresponding to $1 \%$ of the capillary volume, (B) injection of $114 \mathrm{~nL}$, corresponding to $18 \%$ of the capillary volume, (C) injection of $220 \mathrm{~nL}$, corresponding to $35 \%$ of the capillary volume, (D) injection of $352 \mathrm{~nL}$, corresponding to $55 \%$ of the capillary volume. The MS spectra show the comigration of glycopeptides of interest with an autolysis product from bovine trypsin with peptide sequence (pep.seq) QTIASN. PEP illustrates the tryptic peptide sequence EEQYNSTYR. 


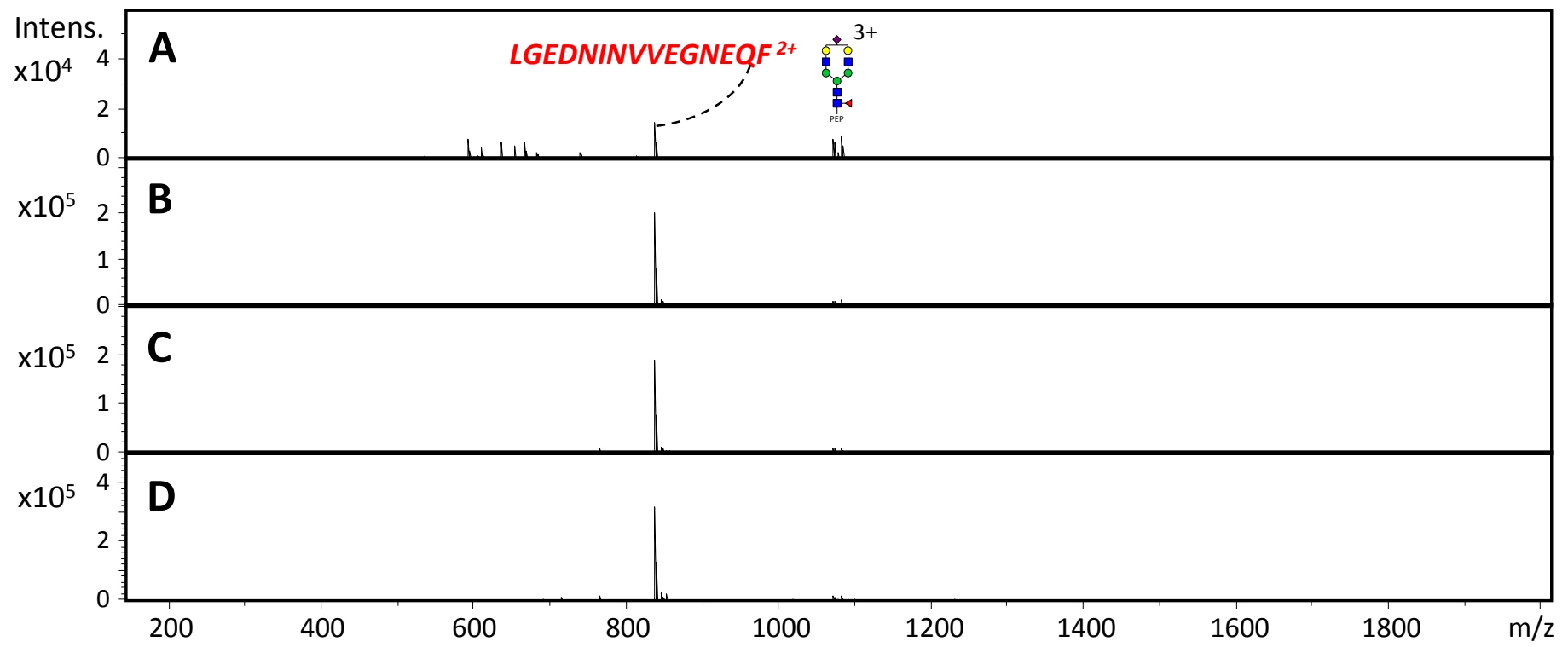

Figure S-18.1. Summed spectra recorded between 22.7 and $27.0 \mathrm{~min}$ corresponding to the migration of the tryptic sialylated Fc $\mathbf{N}$-glycopeptides from polyclonal IgG with different injection volumes using conventional CE-ESI-MS. (A) Injection of $8.8 \mathrm{~nL}$, corresponding to $1 \%$ of the capillary volume, (B) injection of $114 \mathrm{~nL}$, corresponding to $18 \%$ of the capillary volume, (C) injection of $220 \mathrm{~nL}$, corresponding to $35 \%$ of the capillary volume, (D) injection of $352 \mathrm{~nL}$, corresponding to $55 \%$ of the capillary volume. The MS spectra show the comigration of glycopeptides of interest with an autolysis product from bovine trypsin with peptide sequence (pep.seq) LGEDNINVVEGNEQF. PEP illustrates the tryptic peptide sequence EEQYNSTYR.

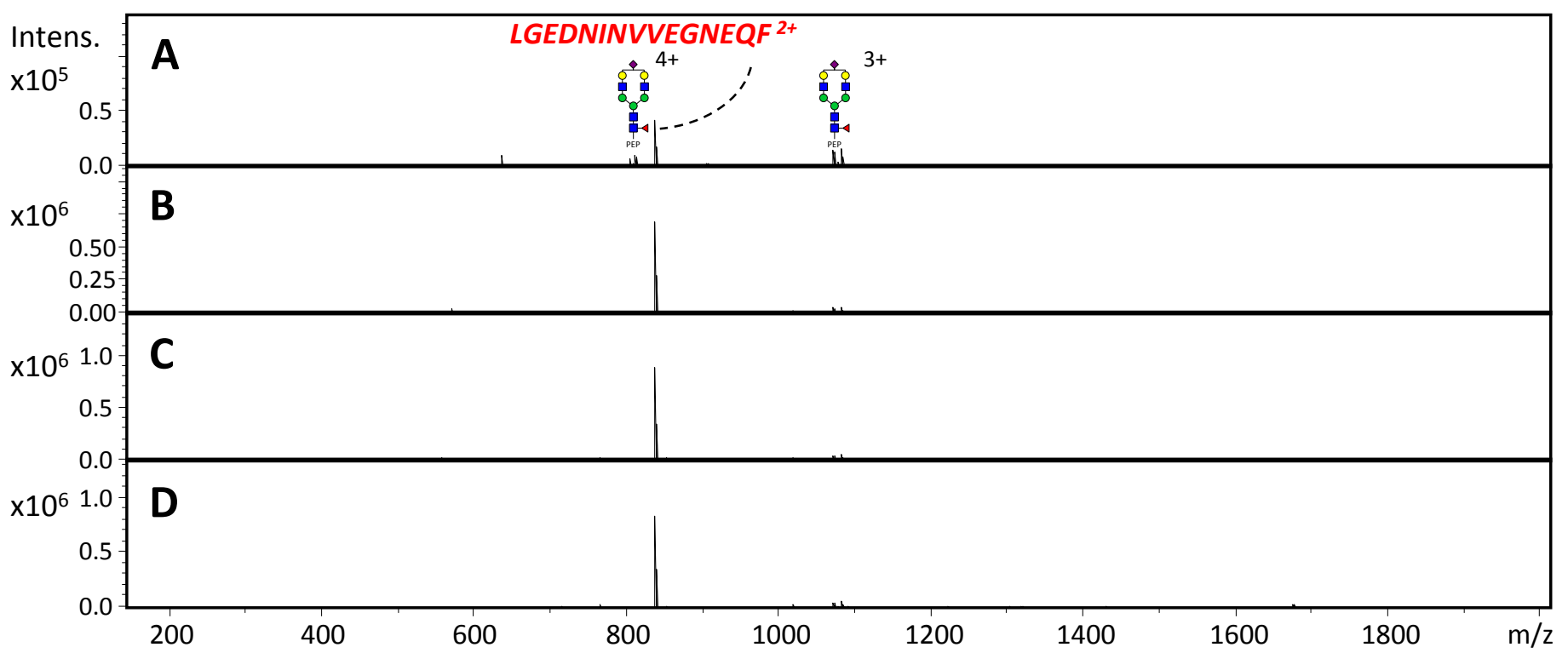

Figure S-18.2. Summed spectra recorded between 22.7 and $\mathbf{2 7 . 0}$ min corresponding to the migration of the tryptic sialylated Fc $\mathbf{N}$-glycopeptides from polyclonal IgG with different injection volumes using CE-ESI-MS with DEN-gas. (A) Injection of $8.8 \mathrm{~nL}$, corresponding to $1 \%$ of the capillary volume, (B) injection of $114 \mathrm{~nL}$, corresponding to $18 \%$ of the capillary volume, (C) injection of $220 \mathrm{~nL}$, corresponding to $35 \%$ of the capillary volume, (D) injection of $352 \mathrm{~nL}$, corresponding to $55 \%$ of the capillary volume. The MS spectra show the comigration of glycopeptides of interest with an autolysis product from bovine trypsin with peptide sequence (pep.seq) LGEDNINVVEGNEQF. PEP illustrates the tryptic peptide sequence EEQYNSTYR. 


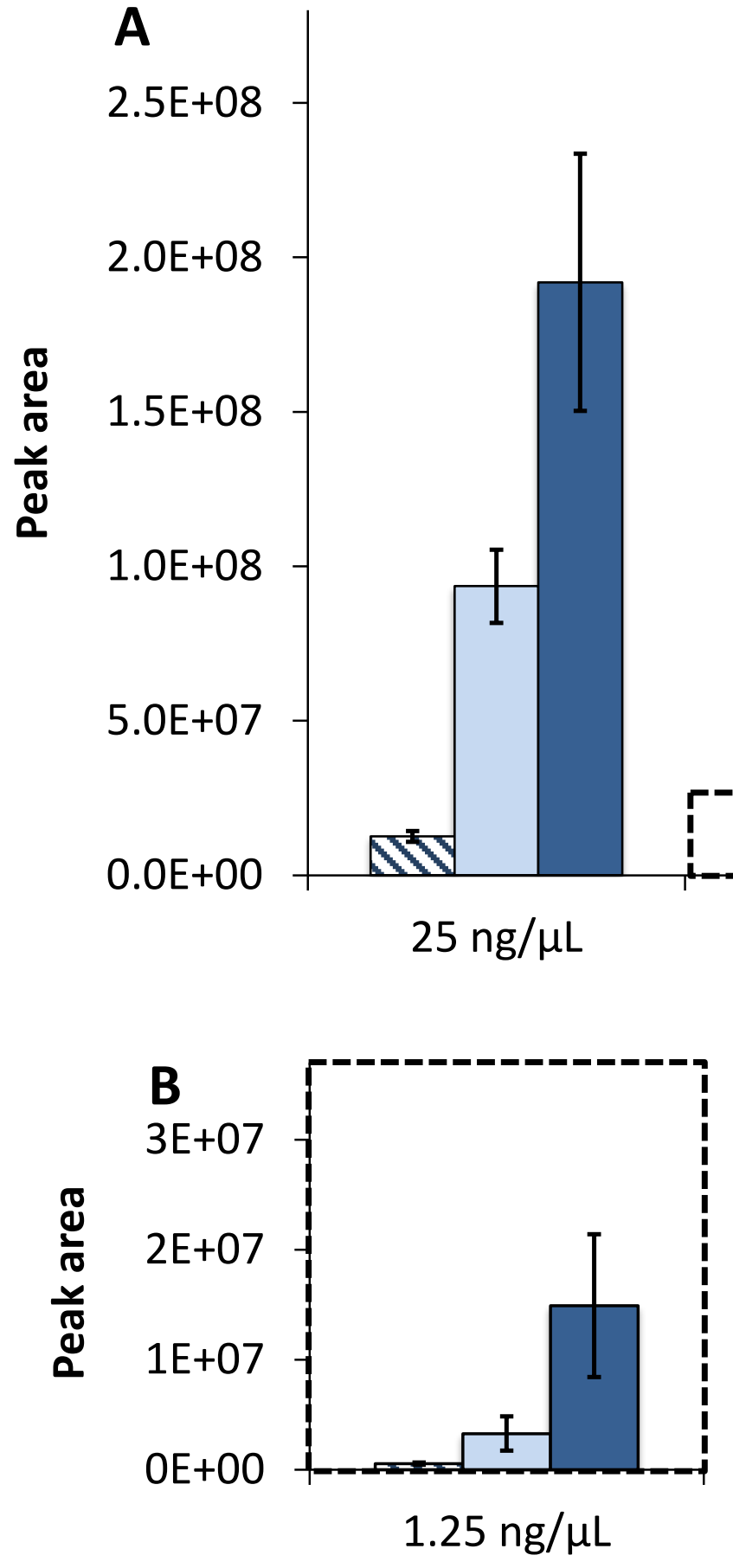

\nano-LC-ESI-MS

$\square$ CE-ESI-MS

$\square$ CE-ESI-MS with DEN-gas

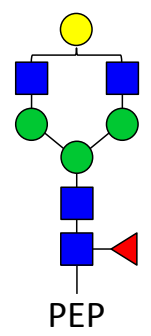

\section{Concentration}

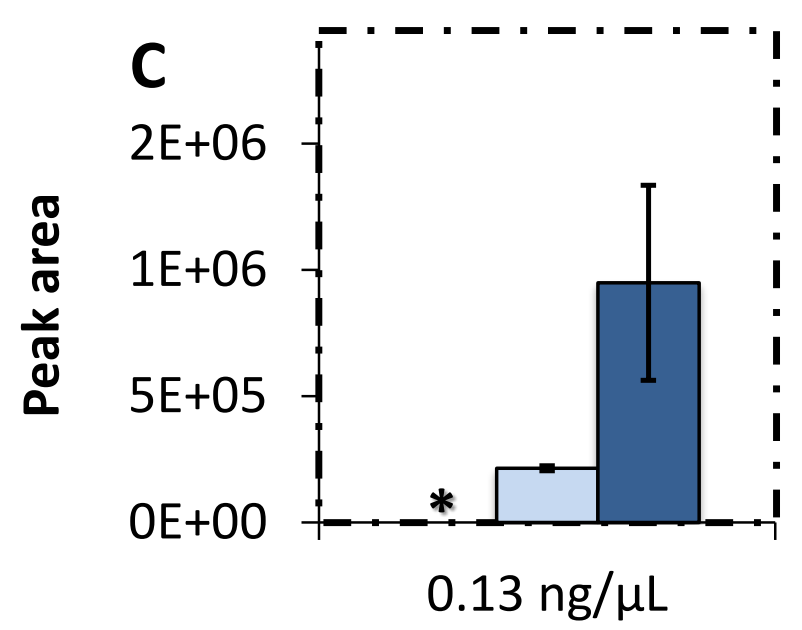

Figure S-19. Peak areas of tryptic Fc $\mathrm{N}$-glycopeptide G1F from the IgGmAb1 obtained with nano-LC-ESI-MS, conventional CE-ESI-MS, and CE-ESI-MS with DEN-gas at different concentrations. Peak areas were measured on EIE of the first three isotopes of doubly, triply and quaternary charged species of glycopeptide G1F. For CE-MS experiments, samples were further diluted with leading electrolyte $(3: 2, v / v)$ prior to injection. (A) Peak areas observed at relatively high, medium, and low concentrations. Magnifications of the middle and low concentration is displayed in (B) Peak areas observed at $1.25 \mathrm{ng} / \mu \mathrm{L}$ and (C) Peak areas observed at $0.13 \mathrm{ng} / \mu \mathrm{L}$. This concentration was below the LOD of nano-LC-ESI-MS and hence G1F was not detected (*). Error bars represent the standard deviation $(N=3)$. The PEP illustrates the tryptic peptide sequence EEQYNSTYR. 

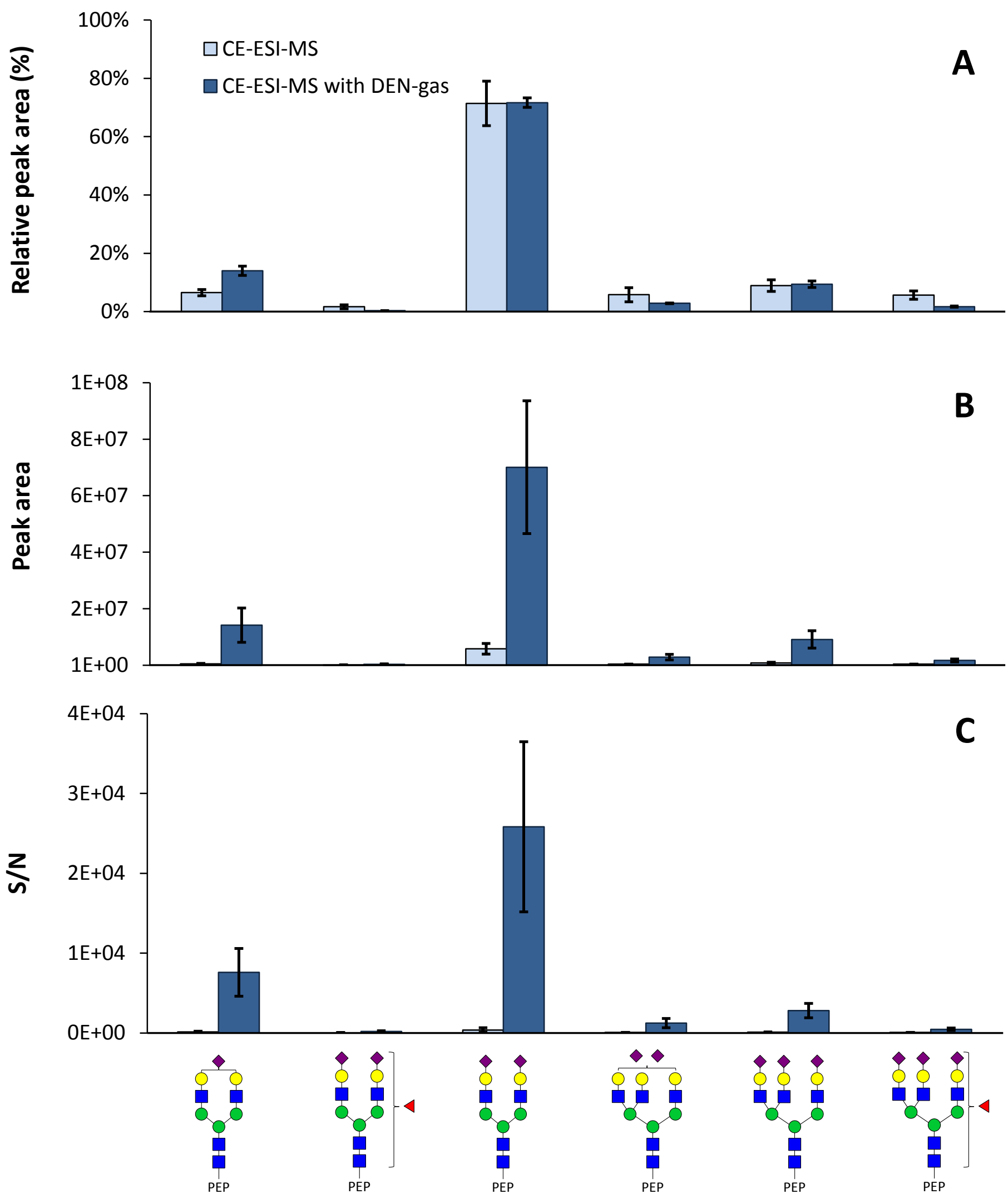

Figure S-20. Differences in peak area and $\mathrm{S} / \mathrm{N}$ observed for the tryptic glycopeptides at glycosylation site $\mathrm{N}_{184}$ from Hp between conventional CE-ESI-MS (light blue) and CE-ESI-MS with DEN-gas setup (dark blue). (A) Relative peak areas, (B) absolute peak areas, (C) S/N ratios. Peak areas and S/N ratios were measured on EIE of the first three isotopes of doubly, triply and quaternary charged species. Error bars represent the standard deviation $(N=3)$. PEP illustrates the tryptic peptide sequence MVSHHNLTTGATLINEQWLLTTAK. 

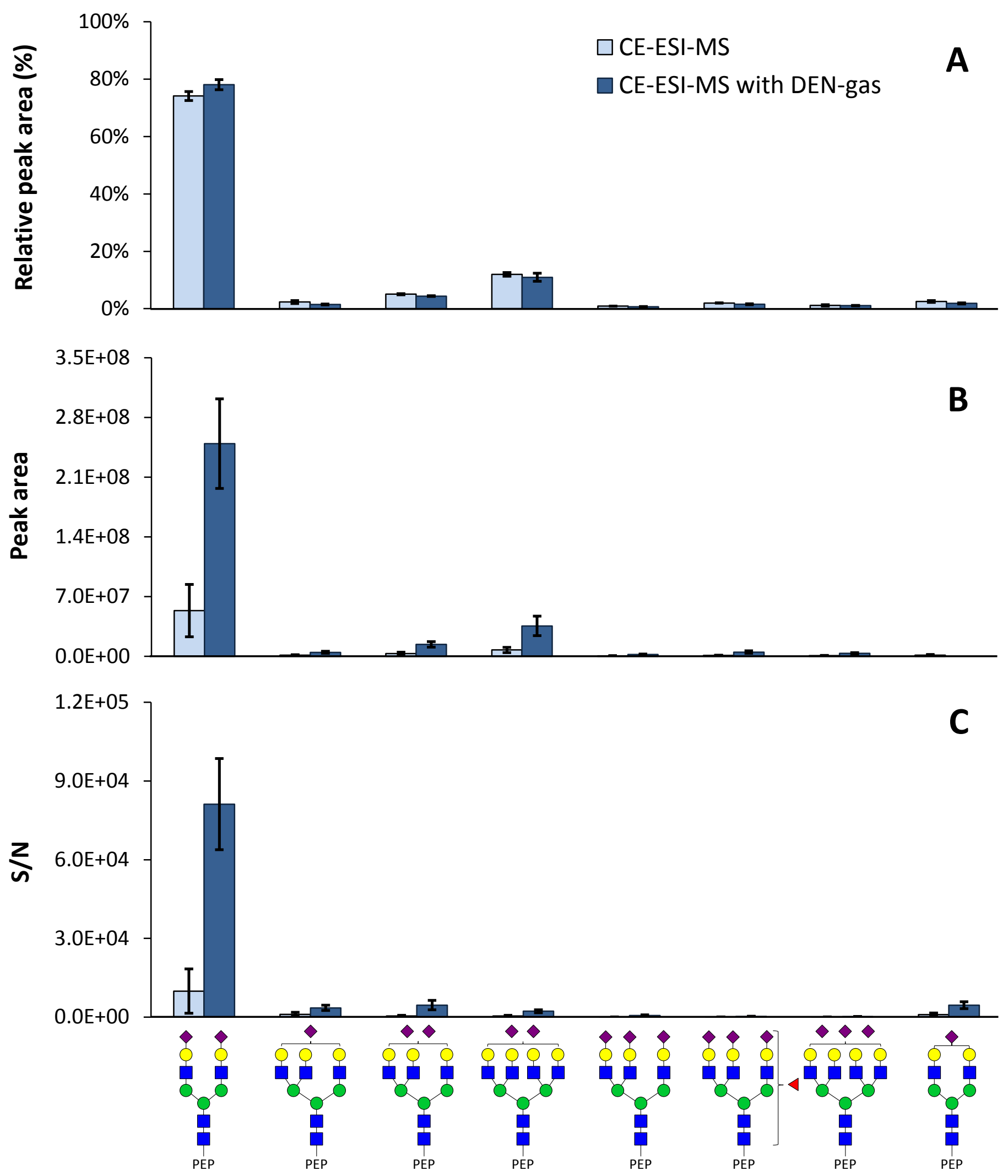

Figure S-21. Differences in peak area and $\mathrm{S} / \mathrm{N}$ observed for the tryptic glycopeptides at glycosylation site $\mathrm{N}_{241}$ from Hp between conventional CE-ESI-MS (light blue) and CE-ESI-MS with DEN-gas setup (dark blue). (A) Relative peak areas, (B) absolute peak areas, (C) S/N ratios. Peak areas and S/N ratios were measured on EIE of the first three isotopes of doubly, triply and quaternary charged species. Error bars represent the standard deviation $(N=3)$. PEP illustrates the tryptic peptide sequence VVLHPNYSQVDIGLIK. 

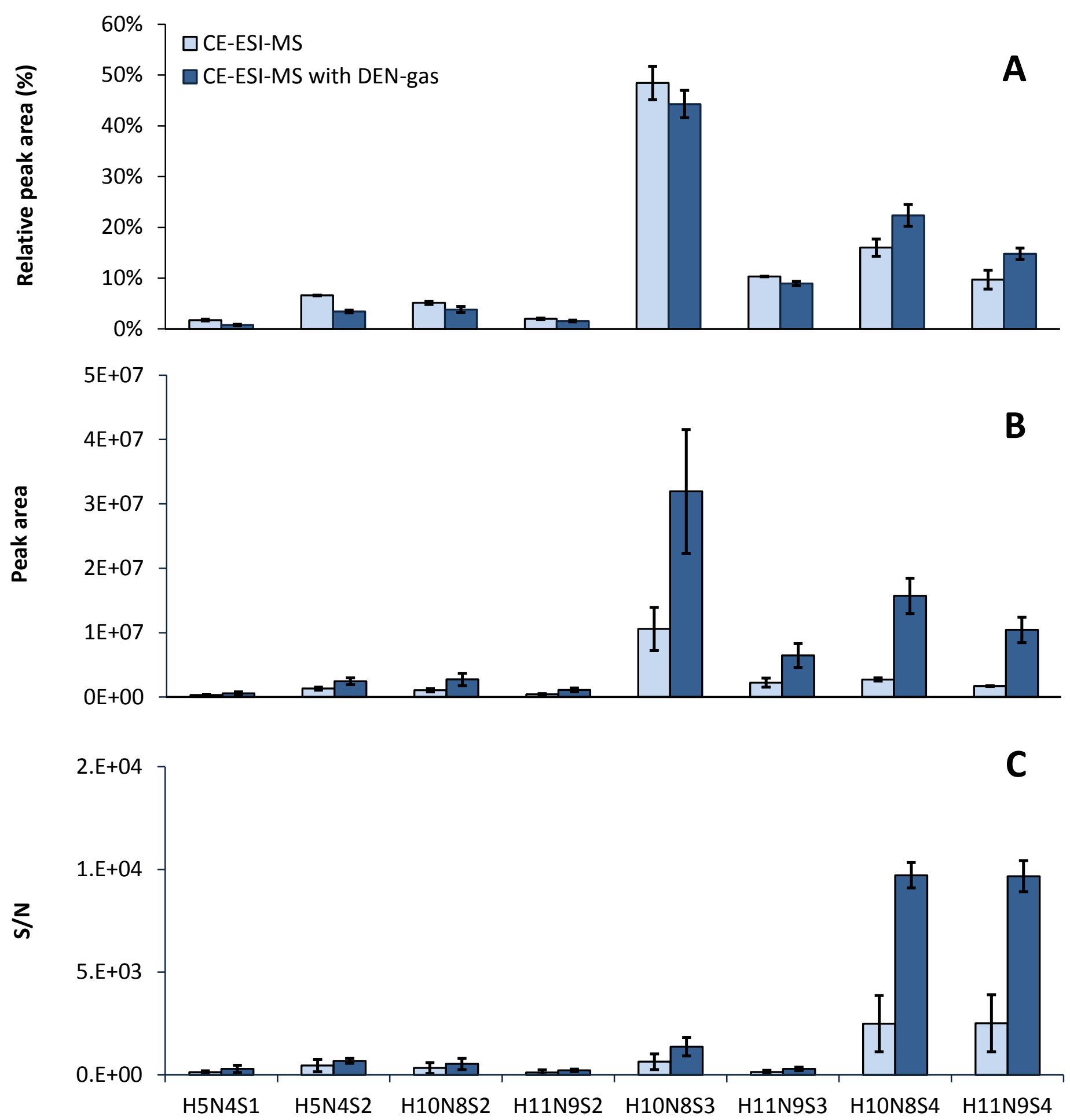

Figure S-22. Differences in peak area and $\mathrm{S} / \mathrm{N}$ observed for the tryptic glycopeptides at glycosylation sites $\mathrm{N}_{207}$ and $\mathrm{N}_{211}$ from $\mathrm{Hp}$ between conventional CE-ESI-MS (light blue) and CE-ESI-MS with DEN-gas setup (dark blue). (A) Relative peak areas, (B) absolute peak areas, (C) $\mathrm{S} / \mathrm{N}$ ratios. Peak areas and $\mathrm{S} / \mathrm{N}$ ratios were measured on EIE of the first three isotopes of doubly, triply and quaternary charged species. Error bars represent the standard deviation $(N=3)$. PEP illustrates the tryptic peptide sequence NLFLNHSENATAK. 

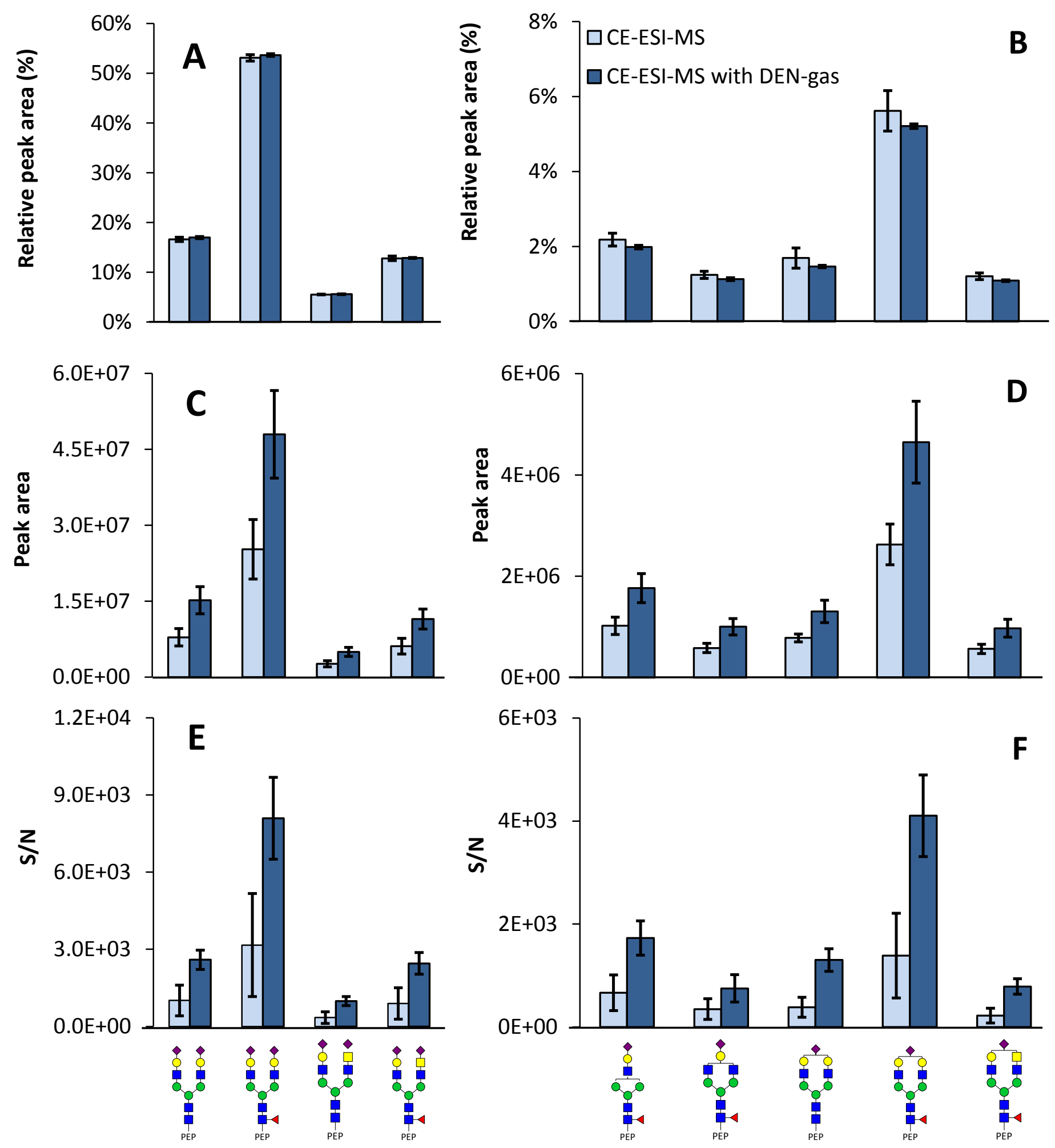

Figure S-23. Differences in peak area and $\mathrm{S} / \mathrm{N}$ observed for high abundant tryptic glycopeptides at glycosylation sites $\mathbf{N}_{69}$ from PSA between conventional CE-ESI-MS (light blue) and CE-ESI-MS with DEN-gas setup (dark blue). (A) Relative peak areas of di-sialylated glycopeptides, (B) relative peak areas of mono-sialylated glycopeptides. (C) Absolute peak areas of di-sialylated glycopeptides, (D) absolute peak areas of mono-sialylated glycopeptides. (E) S/N ratio of di-sialylated glycopeptides, (F) S/N ratio of mono-sialylated glycopeptides. Peak areas and $\mathrm{S} / \mathrm{N}$ ratios were measured on EIE of the first three isotopes of doubly, triply and quaternary charged species. Error bars represent the standard deviation $(N=5)$. PEP illustrates the tryptic peptide sequence NK. 


\section{S-5 REFERENCES}

[1] Selman, M. H.; Hemayatkar, M.; Deelder, A. M.; Wuhrer, M. Analytical chemistry 2011, 83, 2492-2499. 